\title{
Static Grasp Stability Analysis of Multiple Spatial
}

\section{Objects}

\author{
Takayoshi Yamada and Hidehiko Yamamoto \\ Department of Mechanical Engineering, Gifu University, 1-1, Yanagido, Gifu 501-1193, Japan
}

\begin{abstract}
This paper analyzes grasp stability of multiple objects in three dimensions. Every finger is replaced with a translational linear spring model. The stability is formulated by a potential energy stored in the spring. The significant features are provided as follows: The contact surface geometry (metric tensor, curvature, and torsion) is included. Not only rolling contact but also sliding contact is investigated. In the case of rolling contact, partial derivatives of contact position parameters are derived in detail. In the case of sliding contact, novel independent parameters are introduced and their partial derivatives are derived. Finally, grasp force and moment vectors and grasp stiffness matrices are analytically derived. The results are clearer than those of the previous methods. Moreover, the problem on flat surfaces in the previous methods is overcome. Through numerical examples, the effectiveness of our proposed method is demonstrated.
\end{abstract}

Key words: Grasp stability, multiple spatial objects, contact surface geometry, grasp stiffness matrix, translational linear spring model.

\section{Introduction}

Human beings can grasp and manipulate various shaped objects dexterously. Multifingered robot hands have similar potentials because they are created based on human hands. Considering the task that many objects are moved from one place to another place by the hands, and the problem shortening total execution time of the task. One solution is to grasp a single object one by one and to speed up every handling task. Another solution is to grasp multiple objects at the same time and to shorten the number of iterations of the handling task.

In the handling tasks, grasp stability is an important factor. The hands have to maintain stable grasp even if the grasped objects are moved from an initial grasp position under the influence of an external disturbance. The grasp stability is investigated by many researchers. Hanafusa and Asada [1] investigated the stability of grasping a single object. Elastic fingers are replaced by linear spring model, total potential energy stored in

Corresponding author: Takayoshi Yamada, Ph.D., associate professor, research fields: robotic hand, grasp and manipulation, active force sensing, robotic assembly and manufacturing system. the grasp is calculated, and stable grasps are provided from the energy. Nguyen [2] pointed out that the elasticity can be generated by a computer control. They did not consider local curvatures at contact points. Haward and Kumar [3], Rimon and Burdick [4] investigated the effects of the local curvatures. In their methods, the difference of sliding contact and rolling contact was represented by the difference of spring models. One spring model along the contact normal direction was used for the sliding contact and a multiple-springs model was used for the rolling contact. Yamada et al. [5] pointed out that the contact conditions are represented by not the spring models but contact position displacements. The multiple-springs model is used not only for the rolling contact but also for the sliding contact. Grasp stiffness matrices were derived for planar grasps. Yamada et al. [6] investigated spatial grasps. Contact surface geometry (metric tensor, curvature and torsion) was included, and then grasp stiffness matrices are derived.

Harada et al. [7, 8], Yang et al. [9], and Yoshikawa et al. [10] investigated grasping and manipulation of multiple objects. Refs. [7, 8] treated manipulation of 
multiple objects. Refs. [9, 10] discussed a force determination problem for grasping polyhedral objects. They did not investigate the grasp stiffness matrix. Moreover, they did not treat frictionless sliding contact cases.

Yamada et al. [11] investigated grasp stability of multiple planar objects. Yamada et al. [12] extended to grasping of two spatial objects. The conference paper [13] investigated grasping of multiple spatial objects. Refs. [11-13] explored not only rolling contact but also frictionless sliding contact. However, the works have the following problems: The force and moment vectors were not derived clearly. In the case of rolling contact, the formulation has to be refined and derived in detail. In the case of sliding contact, the algorithms fail when either the object surface or the finger surface is flat.

In this paper, we overcome the problems. In Section 2, we describe assumptions and nomenclature. In Section 3, we investigate grasp stability of a single object. Every finger is replaced by a multiple-linear springs model. Contact surface geometry (metric tensor, curvature, and torsion) is included. The potential energy stored in the grasp is formulated. In the case of rolling contact, the rolling contact constraints are provided. The partial derivatives of the constraints are formulated. A contact force vector and stiffness matrix are analytically obtained by partial derivatives of the potential energy. In the case of sliding contact, novel independent parameters are introduced. The parameters are formulated even if the finger surfaces or the object surface are flat. The partial derivatives of the parameters are derived analytically. The contact force vector and stiffness matrix are analytically obtained. In Section 4, we extend to grasping of multiple objects. Independent parameters of objects' displacements are provided. Total potential energy is formulated. Finally, the grasp force and moment vectors and grasp stiffness matrices are derived. In Section 5, effectiveness of our method is demonstrated through numerical examples. In Section 6, we conclude this paper.

\section{Assumptions and Notations}

As shown in Fig. 1, multiple objects are grasped by multi-fingers in three dimensions.

\subsection{Assumptions}

We analyze stability of the grasp in the following assumptions: (A1) The objects and the fingers are rigid bodies; (A2) A single point contact exists between two bodies; (A3) An initial grasp pose (position and orientation) is given; (A4) The surface geometry at each contact point is given; (A5) An infinitesimal pose displacements of the objects occur due to an external disturbance; (A6) Each body slides on the other bodies without friction or rolls on them without slipping; (A7) The relationship between the finger displacement and reaction force is replaced with a $3-D$ translational linear spring model.

From (A1) and (A2), motion of bodies is simplified. From (A3) and (A4), grasp parameters such as the position of contact point, the contact surface geometry, the contact force, and so on are provided. Based on the results of this paper, we will investigate grasp planning problems in our future work. From (A6), roll-slide motion is not considered for simplicity of formulation. From (A7), a virtual spring model is attached to the fingertip as shown in Fig. 1. When the object pose is displaced due to an external disturbance, each finger rolls or slides on the object surface, the spring length changes, and the stored potential energy also changes. From (A5), dynamics is not considered and the grasp stability is analyzed by the first- and the

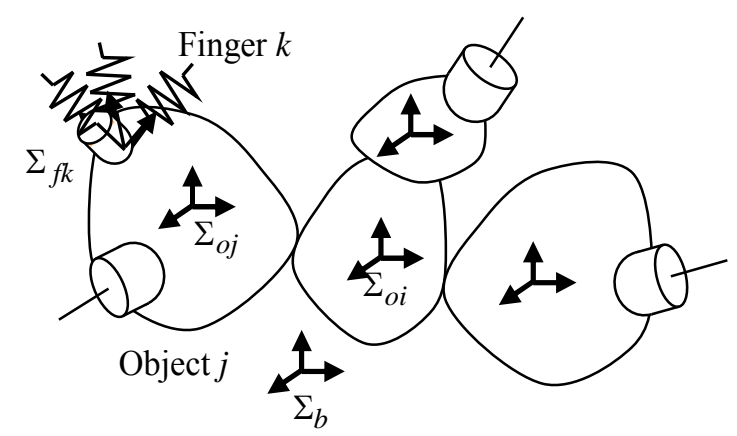

Fig. 1 Multiple objects grasped by multi-fingers. 
second-order partial derivatives of the potential energy at the initial grasp. In our future work, we will attack reduction of the assumptions.

\subsection{Nomenclature}

In this paper, many coordinate frames in Fig. 2 are required because the grasp system is formulated in detail. $\Sigma_{b}$ is a base coordinate frame fixed in the space. $\Sigma_{o i}$ and $\Sigma_{o j}$ are object coordinate frames fixed in the $i$-th and $j$-th objects, respectively. $\Sigma_{C o i j}$, $\Sigma_{C o j i}$, and $\Sigma_{C o j k}$ are current contact frames moving on the objects. $\Sigma_{f k}$ is a finger coordinate frame fixed in the $k$-th finger. $\Sigma_{C f k}$ is a current contact coordinate frame moving on the $k$-th finger, whose $z$ axis is set as its outward normal direction. Frames $\Sigma_{b o i}, \Sigma_{b o j}$ $\Sigma_{b f k}, \Sigma_{L o i j}, \Sigma_{L o j i}, \Sigma_{\text {Lojk }}$ and $\Sigma_{L f k}$ are the initial frames of $\Sigma_{o i}, \Sigma_{o j}, \Sigma_{f k}, \Sigma_{C o i j}, \Sigma_{C o j i}, \Sigma_{C o j k}$, and $\Sigma_{C f k}$, respectively.

Homogeneous transformation matrices and other symbols are shown in Appendix A.

\section{Grasp of a Single Object}

In this section, we concentrate the case that the $k$-th finger maintains contact with the $j$-th object. When the pose of the object is displaced due to an external disturbance, the finger moves on the object surface. We formulate this relation.

\subsection{Pose Displacement of Each Finger}

If the finger is not detached and penetrated with the object, the pose displacement of the finger is obtained

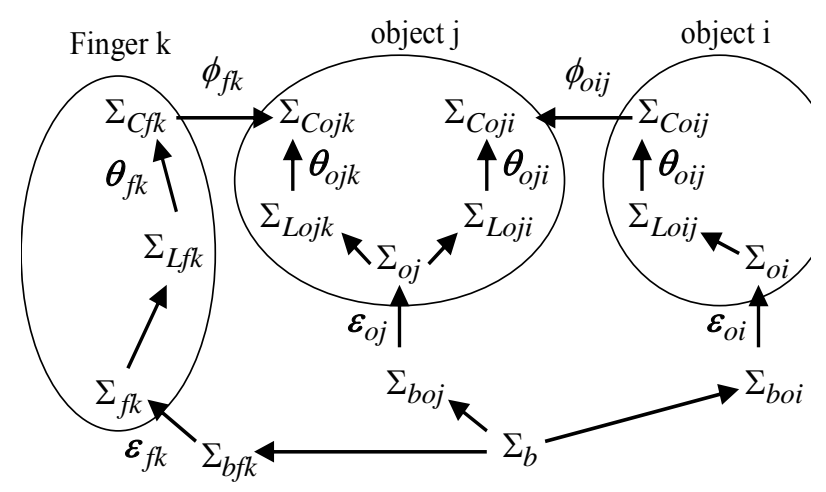

Fig. 2 Coordinate frames used in this analysis. from the following constraint:

$$
\begin{gathered}
{ }^{b} A_{b f k}{ }^{b f k} A_{f k}\left(\boldsymbol{\varepsilon}_{f k}\right)^{f k} A_{L f k} \times \\
{ }^{L f k} A_{C f k}\left(\boldsymbol{\theta}_{f k}\right)^{C f k} A_{\text {Cojk }}\left(\phi_{f k}\right) \\
={ }^{b} A_{b o j}{ }^{b o j} A_{o j}\left(\boldsymbol{\varepsilon}_{o j}\right)^{o j} A_{\text {Lojk }}{ }^{\text {Lojk }} A_{\text {Cojk }}\left(\boldsymbol{\theta}_{o j k}\right)
\end{gathered}
$$

where, $\boldsymbol{\varepsilon}_{o j} \in \mathbb{R}^{6}$ and $\boldsymbol{\varepsilon}_{f k} \in \mathbb{R}^{6}$ are the pose displacements of $\Sigma_{o j}$ and $\Sigma_{f k}$, the symbols $\boldsymbol{\theta}_{o j k} \in \mathbb{R}^{2}$ and $\boldsymbol{\theta}_{f k} \in \mathbb{R}^{2}$ are the contact locations on the $j$-th and $k$-th bodies, and $\phi_{f k} \in \mathbb{R}$ is the spin angle between two bodies. Based on the number of constraints of (7), the parameter $\phi_{f k}$ is assigned not to the object surface side but to the finger surface side. Contact surface geometry is explained in Appendix B.

From Eq. (1), the pose displacement of the finger is obtained by

$$
\begin{gathered}
{ }^{b f k} A_{f k}\left(\boldsymbol{\varepsilon}_{f k}\right)={ }^{b f k} A_{b o j}{ }^{b o j} A_{o j}\left(\boldsymbol{\varepsilon}_{o j}\right) \times \\
{ }^{o j} A_{\text {Lojk }}{ }^{L f k} A_{\text {Lojk }}^{-1}\left(\boldsymbol{\beta}_{f k}\right)^{L f k} A_{f k}
\end{gathered}
$$

where, $\boldsymbol{\beta}_{f k}:=\left[\boldsymbol{\theta}_{f k}^{T}, \phi_{f k}, \boldsymbol{\theta}_{o j k}^{T}\right]^{T}$ and

$$
\begin{gathered}
{ }^{L f k} A_{L o j k}\left(\boldsymbol{\beta}_{f k}\right):={ }^{L f k} A_{C f k}\left(\boldsymbol{\theta}_{f k}\right) \times \\
{ }^{C f k} A_{\text {Cojk }}\left(\phi_{f k}\right){ }^{L o j k} A_{C o j k}^{-1}\left(\boldsymbol{\theta}_{o j k}\right)
\end{gathered}
$$

\subsection{Potential Energy of the Finger}

The finger position displacement is calculated by

$$
\begin{gathered}
\boldsymbol{x}_{f k}\left(\boldsymbol{\varepsilon}_{o j}, \boldsymbol{\beta}_{f k}\right):={ }^{b f k} \boldsymbol{p}_{f k} \\
=I_{34}{ }^{b f k} A_{f k}\left(\boldsymbol{\varepsilon}_{f k}\right) \boldsymbol{v}_{p}
\end{gathered}
$$

The potential energy stored in the finger is given by

$$
\begin{aligned}
U_{j k}\left(\boldsymbol{\varepsilon}_{o j}, \boldsymbol{\beta}_{f k}\right):=\frac{1}{2}\left[\boldsymbol{x}_{f k 0}+\boldsymbol{x}_{f k}\left(\boldsymbol{\varepsilon}_{o j}, \boldsymbol{\beta}_{f k}\right)\right]^{T} \\
\times S_{k}\left[\boldsymbol{x}_{f k 0}+\boldsymbol{x}_{f k}\left(\boldsymbol{\varepsilon}_{o j}, \boldsymbol{\beta}_{f k}\right)\right]
\end{aligned}
$$

From (A7), the spring stiffness of the finger is denoted by $S_{k}:=\operatorname{diag}\left[s_{x k}, s_{y k}, s_{z k}\right] \in \mathbb{R}^{3 \times 3}$. The direction of the stiffness is fixed along the three axes of the frame $\Sigma_{b f k}$. From (A3), the spring is compressed at the initial grasp to generate the initial 
fingertip force $\boldsymbol{f}_{k}:=S_{k} \boldsymbol{x}_{f k 0}$. The first- and second-order partial derivatives of Eq. (5) is given by

$$
\begin{gathered}
{\left[\begin{array}{c}
U_{j k, \varepsilon} \\
U_{j k, \beta}
\end{array}\right]:=\left[\begin{array}{l}
\left.\frac{\partial U_{j k}\left(\boldsymbol{\varepsilon}_{o j}, \boldsymbol{\beta}_{f k}\right)}{\partial \boldsymbol{\varepsilon}_{o j}}\right|_{0} \\
\left.\frac{\partial U_{j k}\left(\boldsymbol{\varepsilon}_{o j}, \boldsymbol{\beta}_{f k}\right)}{\partial \boldsymbol{\beta}_{f k}}\right|_{0}
\end{array}\right]} \\
:\left[\begin{array}{ll}
U_{j k, \varepsilon \varepsilon} & U_{j k, \beta \varepsilon} \\
U_{j k, \varepsilon \beta} & U_{j k, \beta \beta}
\end{array}\right] \\
:=\left[\begin{array}{cl}
\left.\frac{\partial^{2} U_{j k}\left(\varepsilon_{o j}, \boldsymbol{\beta}_{f k}\right)}{\partial \varepsilon_{o j} \partial \boldsymbol{\beta}_{o j}^{T}}\right|_{0} & \left.\frac{\partial^{2} U_{j k}\left(\varepsilon_{o j}, \boldsymbol{\beta}_{f k}\right)}{\partial \boldsymbol{\varepsilon}_{o j} \partial \boldsymbol{\beta}_{f k}^{T}}\right|_{0} \\
\left.\frac{\partial^{2} U_{j k}\left(\varepsilon_{o j}, \boldsymbol{\beta}_{f k}\right)}{\partial \boldsymbol{\beta}_{f k} \partial \varepsilon_{o j}^{T}}\right|_{0} & \left.\frac{\partial^{2} U_{j k}\left(\boldsymbol{\varepsilon}_{o j}, \boldsymbol{\beta}_{f k}\right)}{\partial \boldsymbol{\beta}_{f k} \partial \boldsymbol{\beta}_{f k}^{T}}\right|_{0}
\end{array}\right]
\end{gathered}
$$

The detailed derivations are explained in Appendix C.

\subsection{Fingertip Orientation Constraint}

From (A7), the finger does not rotate. Hence, we have the following finger orientation constraint:

$$
{ }^{b f k} R_{f k}\left(\varepsilon_{o j}, \boldsymbol{\beta}_{f k}\right)=I_{34}{ }^{b f k} A_{f k}\left(\varepsilon_{f k}\right) I_{34}^{T}=I_{3}
$$

The first- and second-order partial derivatives of Eq. (7) are provided in Appendix D. Because three valid constraints are obtained from Eq. (7), two degrees of freedom remain in the parameter $\boldsymbol{\beta}_{f k}$. The remaining degrees are constrained by either the rolling contact explained in section 3.4 or the sliding contact explained in section 3.5.

\subsection{Rolling Contact Finger}

In the case of rolling contact, the relative velocity of two bodies at the contact point at time $t=0$ becomes zero:

$$
I_{24}\left[\left.\hat{V}_{L o j k, L f k}^{b}\left(\boldsymbol{\beta}_{f k}\right)\right|_{0}\right] \boldsymbol{v}_{p}=0_{2 \times 1}
$$

The first- and second-order partial derivatives of Eq. (8) are explained in Appendix D. From Eqs. (7) and (8), the first-order partial derivative of $\boldsymbol{\beta}_{f k}$ is given by the following form:

$$
\begin{gathered}
\boldsymbol{\beta}_{f k, \varepsilon}^{f r}:=\left.\frac{\partial \boldsymbol{\beta}_{f k}^{f r}\left(\boldsymbol{\varepsilon}_{o j}\right)}{\partial \boldsymbol{\varepsilon}_{o j}^{T}}\right|_{0} \\
=\left\{\left[\begin{array}{c}
I_{26} \\
I_{3 \xi}
\end{array}\right] D_{L f k}\right\}^{-1}\left[\begin{array}{c}
0_{2 \times 3} \\
I_{3}
\end{array}\right]{ }^{L f k} R_{o j} I_{3 \xi}
\end{gathered}
$$

The superscript " $f r$ " stands for the rolling contact finger. From Eqs. (7) and (8), the potential energy is reduced to the following form:

$$
U_{j k}^{f r}\left(\boldsymbol{\varepsilon}_{o j}\right):=U_{j k}\left(\boldsymbol{\varepsilon}_{o j}, \boldsymbol{\beta}_{f k}^{f r}\left(\boldsymbol{\varepsilon}_{o j}\right)\right)
$$

The first- and the second-order partial derivatives of Eq. (10) are given by the following form:

$$
\begin{aligned}
& G_{j k}^{f r}:=\left.\frac{\partial U_{j k}^{f r}\left(\boldsymbol{\varepsilon}_{o j}\right)}{\partial \boldsymbol{\varepsilon}_{o j}}\right|_{0}={ }^{o j} W_{L f k}{ }^{L f k} \boldsymbol{f}_{k} \\
& H_{j k}^{f r}:=\left.\frac{\partial^{2} U_{j k}^{f r}\left(\varepsilon_{o j}\right)}{\partial \varepsilon_{o j} \partial \varepsilon_{o j}^{T}}\right|_{0} \\
& =\left[\begin{array}{c}
I_{6} \\
\boldsymbol{\beta}_{f k, \varepsilon}^{f r}
\end{array}\right]^{T}\left[\begin{array}{cc}
U_{j k, \varepsilon \varepsilon} & U_{j k, \beta \varepsilon} \\
U_{j k, \varepsilon \beta} & U_{j k, \beta \beta}
\end{array}\right]\left[\begin{array}{c}
I_{6} \\
\boldsymbol{\beta}_{f k, \varepsilon}^{f r}
\end{array}\right] \\
& +\left[\left.\frac{\partial^{2}\left[U_{j k, \beta}^{T} \beta_{f k}^{f r}\left(\varepsilon_{o j}\right)\right]}{\partial \varepsilon_{o j} \partial \varepsilon_{o j}^{T}}\right|_{0}\right]
\end{aligned}
$$

The detailed derivations are shown in Appendix E. The force vector ${ }^{L f k} \boldsymbol{f}_{k}$ is represented in the frame $\Sigma_{L f k}$.

$$
\begin{gathered}
{ }^{L f k} \boldsymbol{f}_{k}=\left[\begin{array}{ll}
{ }^{L f k} f_{x k} \\
{ }^{L f k} f_{y k} \\
{ }^{L f k} f_{z k}
\end{array}\right]:={ }^{L f k} R_{b f k} \boldsymbol{f}_{k} \\
{ }^{L f k} f_{z k} \leq 0
\end{gathered}
$$

The force is a reaction force from the object to the finger. The $z$-axis direction of $\Sigma_{L f k}$ is the outward normal direction of the finger surface. Hence the $z$ component of ${ }^{L f k} \boldsymbol{f}_{k}$ is negative.

As shown in Eqs. (9) and (11), $\boldsymbol{\beta}_{f k, \varepsilon}^{f r}$ and $G_{j k}^{f r}$ are obtained clearly although they were complicated in Refs. [6, 12, 13].

\subsection{Sliding Contact Finger}

\subsubsection{Independent Parameters}

The independent parameters of Eq. (7) are defined as $\gamma_{k} \in \mathbb{R}^{2}$. The contact displacement parameters can be represented as $\boldsymbol{\beta}_{f k}^{r c}\left(\boldsymbol{\varepsilon}_{o j}, \boldsymbol{\gamma}_{k}\right)$, where the superscript 
"rc" means the finger's rotation constraint. We define the selection matrices $I_{\gamma k} \in \mathbb{R}^{2 \times 5}$ and $I_{r c k} \in \mathbb{R}^{3 \times 5}$ satisfying the following conditions:

$$
\begin{gathered}
\boldsymbol{\gamma}_{k}=I_{\gamma k} \boldsymbol{\beta}_{f k}^{r c}\left(\boldsymbol{\varepsilon}_{o j}, \boldsymbol{\gamma}_{k}\right) \\
I_{\gamma k}^{T} I_{\gamma k}+I_{r c k}^{T} I_{r c k}=I_{5}, \quad I_{\gamma k} I_{\gamma k}^{T}=I_{2} \\
I_{r c k} I_{r c k}^{T}=I_{3}, \quad I_{\gamma k} I_{r c k}^{T}=0_{2 \times 3}
\end{gathered}
$$

The displacements $\boldsymbol{\varepsilon}_{o j}$ and $\boldsymbol{\gamma}_{k}$ satisfy the following constraint:

$$
{ }^{b f k} R_{f k}\left(\boldsymbol{\varepsilon}_{o j}, \boldsymbol{\beta}_{f k}^{r c}\left(\boldsymbol{\varepsilon}_{o j}, \boldsymbol{\gamma}_{k}\right)\right)=I_{3}
$$

From the first-order partial derivative of Eq. (14), we have

$$
\begin{gathered}
\boldsymbol{\beta}_{f k, \varepsilon}^{r c}:=\left.\frac{\partial \boldsymbol{\beta}_{f k}^{r c}\left(\boldsymbol{\varepsilon}_{o j}, \boldsymbol{\gamma}_{k}\right)}{\partial \boldsymbol{\varepsilon}_{o j}^{T}}\right|_{0} \\
=I_{r c k}^{T} P_{d k}^{-1 L f k} R_{o j} I_{3 \xi} \\
\boldsymbol{\beta}_{f k, \gamma}^{r c}:=\left.\frac{\partial \boldsymbol{\beta}_{f k}^{r c}\left(\boldsymbol{\varepsilon}_{o j}, \boldsymbol{\gamma}_{k}\right)}{\partial \boldsymbol{\gamma}_{k}^{T}}\right|_{0} \\
=I_{\gamma k}^{T}-I_{r c k}^{T} P_{d k}^{-1} I_{3 \xi} D_{L f k} I_{\gamma k}^{T}
\end{gathered}
$$

where,

$$
P_{d k}:=I_{3 \xi} D_{L f k} I_{r c k}^{T} \in \mathbb{R}^{3 \times 3}
$$

The detailed derivations of Eq. (15) are shown in Appendix F. The matrices $I_{\gamma k}$ and $I_{r c k}$ can be selected from several types.

\subsubsection{Case of Ref. [6]}

If $\boldsymbol{\theta}_{f k}$ is selected as $\boldsymbol{\gamma}_{k}$, the selection matrices are given as the following form:

$$
I_{\gamma k}=\left[\begin{array}{ll}
I_{2} & 0_{2 \times 3}
\end{array}\right], I_{r c k}=\left[\begin{array}{ll}
0_{3 \times 2} & I_{3}
\end{array}\right]
$$

From Eqs. (17) and (77), we have

$$
P_{d k}=\left[\begin{array}{cc}
0_{2 \times 1} & -{ }^{L f k} \tilde{R}_{L o j k} \Omega K_{o j k} M_{o j k} \\
1 & T_{o j k} M_{o j k}
\end{array}\right]
$$

If the object surface is flat, $K_{o j k}$ becomes a zero matrix and $P_{d k}$ becomes singular. Hence, Eq. (17) is inapplicable to the case that the object surface is flat.

\subsubsection{Case of Refs. $[12,13]$}

If $\boldsymbol{\theta}_{o j k}$ is selected as $\boldsymbol{\gamma}_{k}$, the matrices are given as the following form:

$$
I_{\gamma k}=\left[\begin{array}{ll}
0_{2 \times 3} & I_{2}
\end{array}\right], I_{r c k}=\left[\begin{array}{ll}
I_{3} & 0_{3 \times 2}
\end{array}\right] .
$$

From Eqs. (19) and (77), we have

$$
P_{d k}=\left[\begin{array}{cc}
\Omega K_{f k} M_{f k} & 0_{2 \times 1} \\
T_{f k} M_{f k} & 1
\end{array}\right]
$$

If the finger surface is flat, $K_{f k}$ becomes a zero matrix and $P_{d k}$ becomes singular. Hence, Eq. (19) is inapplicable to the case that the finger surface is flat.

\subsubsection{Novel Independent Parameters}

The relative motion displacement between $\Sigma_{L o j k}$ and $\Sigma_{L f k}$ is selected as $\gamma_{k}$. The selection matrices are provided as the following form:

$$
\begin{gathered}
I_{\gamma k}=P_{\gamma k} I_{26} D_{L f k} \\
=P_{\gamma k}\left[\begin{array}{lll}
M_{f k} & 0_{2 \times 1} & -\widetilde{M}_{o j k}
\end{array}\right] \\
I_{r c k}=\left[\begin{array}{cc}
P_{\gamma k}^{-1} & 0_{2 \times 1} \\
0_{1 \times 2} & 1
\end{array}\right]\left[\begin{array}{ccc}
M_{f k}^{-T} & 0_{2 \times 1} & \widetilde{M}_{o j k}^{-T} \\
0_{1 \times 2} & 1 & 0_{1 \times 2}
\end{array}\right]
\end{gathered}
$$

where the matrix $P_{\gamma k} \in \mathbb{R}^{2 \times 2}$ has to satisfy

$$
P_{\gamma k}^{T} P_{\gamma k}=\left[M_{f k} M_{f k}^{T}+\widetilde{M}_{o j k} \widetilde{M}_{o j k}^{T}\right]^{-1}
$$

Eq. (22) satisfies the other conditions described in Eq. (13). From Eqs. (21) and (77), we have

$$
P_{d k}=\left[\begin{array}{cc}
\Omega\left[K_{f k}+\widetilde{K}_{o j k}\right] P_{\gamma k}^{-T} & 0_{2 \times 1} \\
{\left[T_{f k}+\widetilde{T}_{o j k}\right] P_{\gamma k}^{-T}} & 1
\end{array}\right]
$$

where

$$
\begin{gathered}
\widetilde{M}_{o j k}:={ }^{L f k} \tilde{R}_{L o j k} M_{o j k}, \\
\widetilde{K}_{o j k}:={ }^{L f k} \tilde{R}_{L o j k} K_{o j k}{ }^{L f k} \tilde{R}_{L o j k}^{-1} \\
\widetilde{T}_{o j k}:=T_{o j k}{ }^{L f k} \tilde{R}_{L o j k}^{-1}
\end{gathered}
$$

From (A2), we have the following condition:

$$
K_{f k}+\widetilde{K}_{o j k}>0_{2 \times 2}
$$

Hence, $P_{d k}$ is nonsingular.

$$
\operatorname{det} P_{d k}=\frac{\operatorname{det}\left[K_{f k}+\widetilde{K}_{o j k}\right]}{\operatorname{det} P_{\gamma k}} \neq 0
$$

Consequently, this paper uses Eq. (21).

\subsubsection{Potential Energy}

Considering Eq. (13), the potential energy is reduced to the following form:

$$
U_{j k}^{r c}\left(\boldsymbol{\varepsilon}_{o j}, \boldsymbol{\gamma}_{k}\right):=U_{j k}\left(\boldsymbol{\varepsilon}_{o j}, \boldsymbol{\beta}_{f k}^{r c}\left(\boldsymbol{\varepsilon}_{o j}, \boldsymbol{\gamma}_{k}\right)\right)
$$

The first- and second-order partial derivatives of Eq. (27) are given by the following form: 


$$
\begin{gathered}
{\left[\begin{array}{c}
U_{j k, \varepsilon}^{r c} \\
U_{j k, \gamma}^{r c}
\end{array}\right]:=\left[\begin{array}{l}
\left.\frac{\partial U_{j k}^{r c}\left(\boldsymbol{\varepsilon}_{o j}, \boldsymbol{\gamma}_{k}\right)}{\partial \varepsilon_{o j}}\right|_{0} \\
\left.\frac{\partial U_{j k}^{r c}\left(\boldsymbol{\varepsilon}_{o j}, \boldsymbol{\gamma}_{k}\right)}{\partial \boldsymbol{\gamma}_{k}}\right|_{0}
\end{array}\right]:} \\
{\left[\begin{array}{ll}
U_{j k, \varepsilon \varepsilon}^{r c} & U_{j k, \gamma \varepsilon}^{r c} \\
U_{j k, \varepsilon \gamma}^{r c} & U_{j k, \gamma \gamma}^{r c}
\end{array}\right]:} \\
=\left[\begin{array}{ll}
\left.\frac{\partial^{2} U_{j k}^{r c}\left(\varepsilon_{o j}, \gamma_{k}\right)}{\partial \varepsilon_{o j} \partial \varepsilon_{o j}^{T}}\right|_{0} & \left.\frac{\partial^{2} U_{j k}^{r c}\left(\varepsilon_{o j}, \gamma_{k}\right)}{\partial \varepsilon_{o j} \partial \gamma_{k}^{T}}\right|_{0} \\
\left.\frac{\partial^{2} U_{j k}^{r c}\left(\varepsilon_{o j}, \gamma_{k}\right)}{\partial \gamma_{k} \partial \varepsilon_{o j}^{T}}\right|_{0} & \left.\frac{\partial^{2} U_{j k}^{r c}\left(\varepsilon_{o j}, \gamma_{k}\right)}{\partial \gamma_{k} \partial \gamma_{k}^{T}}\right|_{0}
\end{array}\right]
\end{gathered}
$$

The derivatives are provided in Appendix G. In the case of sliding contact, each fingertip moves on the object surface to locally minimize its potential energy. Hence, we have the following constraints:

$$
\boldsymbol{h}_{j k}^{r c}\left(\boldsymbol{\varepsilon}_{o j}, \boldsymbol{\gamma}_{k}\right):=\frac{\partial U_{j k}^{r c}\left(\varepsilon_{o j}, \gamma_{k}\right)}{\partial \gamma_{k}}=0_{2 \times 1}
$$

and

$$
\frac{\partial^{2} U_{j k}^{r c}\left(\varepsilon_{o j}, \gamma_{k}\right)}{\partial \gamma_{k} \partial \gamma_{k}^{T}}>0_{2 \times 2}
$$

At the initial condition of Eq. (29), we have $U_{j k, \gamma}^{r c}=0_{2 \times 1}$. From Appendix H, we have

$$
{ }^{L f k} \boldsymbol{f}_{k}={ }^{L f k} f_{z k} \boldsymbol{u}_{3}
$$

From the initial condition of Eq. (30), we have

$$
U_{j k, \gamma \gamma}^{r c}=\left[\boldsymbol{\beta}_{f k, \gamma}^{r c}\right]^{T} U_{j k, \beta \beta} \boldsymbol{\beta}_{f k, \gamma}^{r c}>0_{2 \times 2}
$$

This positive definiteness provides a stability condition of the finger. From Eq. (29), the parameter $\boldsymbol{\gamma}_{k}$ is given by a function of $\boldsymbol{\varepsilon}_{o j}$. We have the following form:

$$
\boldsymbol{h}_{j k}^{r c}\left(\boldsymbol{\varepsilon}_{o j}, \boldsymbol{\gamma}_{k}^{f s}\left(\boldsymbol{\varepsilon}_{o j}\right)\right)=0_{2 \times 1}
$$

The superscript "fs" means the sliding contact finger. The potential energy is reduced to the following form:

$$
U_{j k}^{f s}\left(\boldsymbol{\varepsilon}_{o j}\right):=U_{j k}^{r c}\left(\boldsymbol{\varepsilon}_{o j}, \boldsymbol{\gamma}_{k}^{f s}\left(\boldsymbol{\varepsilon}_{o j}\right)\right)
$$

From the partial derivative of Eq. (33), we have

$$
\begin{gathered}
\gamma_{k, \varepsilon}^{f s}:=\left.\frac{\partial \gamma_{k}^{f s}\left(\varepsilon_{o j}\right)}{\partial \varepsilon_{o j}^{T}}\right|_{0} \\
=-\left[U_{j k, \gamma \gamma}^{r c}\right]^{-1} U_{j k, \varepsilon \gamma}^{r c} \in \mathbb{R}^{2 \times 6}
\end{gathered}
$$

As shown in Appendix $H$, the first- and second-order partial derivatives of Eq. (34) are given by

$$
\begin{gathered}
G_{j k}^{f s}:=\left.\frac{\partial U_{j k}^{f s}\left(\boldsymbol{\varepsilon}_{o j}\right)}{\partial \boldsymbol{\varepsilon}_{o j}}\right|_{0} \\
={ }^{o j} W_{L f k}{ }^{L f k} \boldsymbol{f}_{k}={ }^{o j} W_{L f k} \boldsymbol{u}_{3}{ }^{L f k} f_{z k} \\
H_{j k}^{f s}:=\left.\frac{\partial^{2} U_{j k}^{f s}\left(\boldsymbol{\varepsilon}_{o j}\right)}{\partial \boldsymbol{\varepsilon}_{o j} \partial \boldsymbol{\varepsilon}_{o j}^{T}}\right|_{0} \\
=U_{j k, \varepsilon \varepsilon}^{r c}-U_{j k, \gamma \varepsilon}^{r c}\left[U_{j k, \gamma \gamma}^{r c}\right]^{-1} U_{j k, \varepsilon \gamma}^{r c}
\end{gathered}
$$

As shown in Eq. (36), $G_{j k}^{f s}$ and $H_{j k}^{f s}$ are more clearly obtained than those of the previous methods $[6$, 12, 13]. Moreover, the problem on the surface curvature has been overcome.

\subsection{Total Energy of the Single Object}

When the object is grasped by $n_{k}$ fingers, the potential energy is represented by the following form:

$$
U_{j}\left(\varepsilon_{o j}\right):=U_{e j}\left(\varepsilon_{o j}\right)+\sum_{k=1}^{n_{k}} U_{f k}^{f c}\left(\varepsilon_{o j}\right)
$$

The energy $U_{e j}\left(\boldsymbol{\varepsilon}_{o j}\right)$ is given by an external force. The first- and second-order partial derivatives of $U_{e j}$ are shown in Appendix I.

\section{Grasp of Multiple Objects}

When the $j$-th object is not detached and penetrated with the $i$-th object, the pose displacement of the object is obtained from the following constraint:

$$
\begin{gathered}
{ }^{b} A_{b o i}{ }^{b o i} A_{o i}\left(\varepsilon_{o i}\right){ }^{o i} A_{L o i j} \times \\
{ }^{L o i j} A_{C o i j}\left(\boldsymbol{\theta}_{o i j}\right){ }^{\text {Coij }} A_{\text {Coji }}\left(\phi_{o i j}\right) \\
={ }^{b} A_{b o j}{ }^{b o j} A_{o j}\left(\varepsilon_{o j}\right)^{o j} A_{L o j i}{ }^{L o j i} A_{C o j i}\left(\boldsymbol{\theta}_{o j i}\right)
\end{gathered}
$$

Based on the number of constraints of Eq. (46), the parameter $\phi_{o i j}$ is assigned not to the $j$-th object side but to the $i$-th object side. The pose of the $j$-th object is 
given by

$$
\begin{gathered}
{ }^{b o j} A_{o j}\left(\boldsymbol{\varepsilon}_{o j}\right)={ }^{b o j} A_{b o i}{ }^{b o i} A_{o i}\left(\boldsymbol{\varepsilon}_{o i}\right) \times \\
{ }^{o i} A_{\text {Loij }}{ }^{\text {Loij }} A_{\text {Loji }}\left(\boldsymbol{\beta}_{o i j}\right)^{\text {Loji }} A_{o j}
\end{gathered}
$$

where, $\boldsymbol{\beta}_{o i j}:=\left[\boldsymbol{\theta}_{o i j}^{T}, \phi_{o i j}, \boldsymbol{\theta}_{o j i}^{T}\right]^{T}$ and

$$
\begin{gathered}
{ }^{\text {Loij }} A_{\text {Loji }}\left(\boldsymbol{\beta}_{o i j}\right):={ }^{\text {Loij }} A_{\text {Coij }}\left(\boldsymbol{\theta}_{o i j}\right) \times \\
{ }^{C o i j} A_{\text {Coji }}\left(\phi_{o i j}\right){ }^{L o j i} A_{C o j i}^{-1}\left(\boldsymbol{\theta}_{o j i}\right)
\end{gathered}
$$

\subsection{Grasp Stiffness Matrix with Sliding Contact}

Considering Eq. (39), the potential energy of Eq. (37) is reduced to

$$
U_{i j}^{o S}\left(\boldsymbol{\varepsilon}_{o i}, \boldsymbol{\beta}_{o i j}\right):=U_{j}\left(\boldsymbol{\varepsilon}_{o j}\left(\boldsymbol{\varepsilon}_{o i}, \boldsymbol{\beta}_{o i j}\right)\right)
$$

The first- and the second-order partial derivatives of Eq. (41) is described in Appendix J. The superscript "os" is used for representing the sliding contact between the objects. The total potential energy of the grasp is given by

$$
U^{o s}\left(\boldsymbol{\alpha}^{o s}\right):=U_{i}\left(\boldsymbol{\varepsilon}_{o i}\right)+\sum_{j=1}^{n_{j}} U_{i j}^{o s}\left(\boldsymbol{\varepsilon}_{o i}, \boldsymbol{\beta}_{o i j}\right)
$$

where,

$$
\boldsymbol{\alpha}^{o s}:=\left[\boldsymbol{\varepsilon}_{o i}^{T}, \boldsymbol{\beta}_{o i 1}^{T}, \boldsymbol{\beta}_{o i 2}^{T}, \cdots, \boldsymbol{\beta}_{o i n_{j}}^{T}\right]^{T} \in \mathbb{R}^{6+5 n_{j}}
$$

The energy $U_{i}\left(\varepsilon_{o i}\right)$ is given in a similar manner of $U_{j}\left(\varepsilon_{o j}\right)$ shown in Eq. (37). The first- and the second-order partial derivatives of Eq. (42) are given by

$$
\begin{aligned}
& G^{o s}:=\frac{\partial U^{o s}\left(\boldsymbol{\alpha}^{o s}\right)}{\partial \boldsymbol{\alpha}^{o S}}=\left[\begin{array}{c}
U_{i, \varepsilon}+\sum_{j=1}^{n_{j}} U_{i j, \varepsilon}^{o s} \\
U_{i 1, \beta}^{o S} \\
\vdots \\
U_{i n_{j}, \beta}^{o S}
\end{array}\right], \\
& H^{o s}:=\frac{\partial^{2} U^{o s}\left(\boldsymbol{\alpha}^{o s}\right)}{\partial \boldsymbol{\alpha}^{o s}\left[\partial \boldsymbol{\alpha}^{o s}\right]^{T}} \\
& =\left[\begin{array}{cccc}
U_{i, \varepsilon \varepsilon}+\sum_{j=1}^{n_{j}} U_{i j, \varepsilon \varepsilon}^{o S} & U_{i 1, \beta \varepsilon}^{o S} & \ldots & U_{i n_{j}, \beta \varepsilon}^{o S} \\
U_{i 1, \varepsilon \beta}^{o S} & U_{i 1, \beta \beta}^{o S} & \ldots & 0_{5 \times 5} \\
\vdots & \vdots & \ddots & \vdots \\
U_{i n_{j}, \varepsilon \beta}^{o S} & 0_{5 \times 5} & \ldots & U_{i n_{j}, \beta \beta}^{o S}
\end{array}\right] \text {, }
\end{aligned}
$$

where,

$$
\begin{aligned}
& {\left[\begin{array}{c}
U_{i j, \varepsilon}^{o S} \\
U_{i j, \beta}^{o S}
\end{array}\right]:=\left[\begin{array}{l}
\left.\frac{\partial U_{i j}^{o S}\left(\boldsymbol{\varepsilon}_{o i}, \boldsymbol{\beta}_{o i j}\right)}{\partial \boldsymbol{\varepsilon}_{o i}}\right|_{0} \\
\left.\frac{\partial U_{i j}^{o S}\left(\boldsymbol{\varepsilon}_{o i}, \boldsymbol{\beta}_{o i j}\right)}{\partial \boldsymbol{\beta}_{o i j}}\right|_{0}
\end{array}\right]} \\
& =\left[{ }^{b o j} B_{b o i} \quad{ }^{b o j} B_{L o i j} D_{L o i j}\right]^{T} U_{j, \varepsilon}, \\
& {\left[\begin{array}{ll}
U_{i j, \varepsilon \varepsilon}^{o s} & U_{i j, \beta \varepsilon}^{o s} \\
U_{i j, \varepsilon \beta}^{o s} & U_{i j, \beta \beta}^{o s}
\end{array}\right]} \\
& :=\left[\begin{array}{ll}
\left.\frac{\partial^{2} U_{i j}^{o S}\left(\varepsilon_{o i} \boldsymbol{\beta}_{o i j}\right)}{\partial \varepsilon_{o i} \partial \varepsilon_{o i}^{T}}\right|_{0} & \left.\frac{\partial^{2} U_{i j}^{o S}\left(\varepsilon_{o i}, \boldsymbol{\beta}_{o i j}\right)}{\partial \varepsilon_{o i} \partial \boldsymbol{\beta}_{o i j}^{T}}\right|_{0} \\
\left.\frac{\partial^{2} U_{i j}^{o S}\left(\varepsilon_{o i} \boldsymbol{\beta}_{o i j}\right)}{\partial \boldsymbol{\beta}_{o i j} \partial \varepsilon_{o i}^{T}}\right|_{0} & \left.\frac{\partial^{2} U_{i j}^{o s}\left(\varepsilon_{o i}, \boldsymbol{\beta}_{o i j}\right)}{\partial \boldsymbol{\beta}_{o i j} \partial \boldsymbol{\beta}_{o i j}^{T}}\right|_{0}
\end{array}\right]
\end{aligned}
$$

The vector $G^{o s}$ is a grasp force and moment vector, and the matrix $H^{o s}$ is a grasp stiffness matrix. The grasp stability is evaluated by the eigenvalues of $H^{o s}$ when $G^{o s}$ is zero (force and moment equilibrium). The directions of object displacements are obtained from the corresponding eigenvectors.

\subsection{Grasp Stiffness Matrix with Rolling Contact}

In the case of rolling contact between objects, each object rolls on other objects. Hence, we have the following constraints.

$$
I_{24}\left[\left.\widehat{V}_{L o j i, L o i j}^{b}\left(\boldsymbol{\beta}_{o i j}\right)\right|_{0}\right] \boldsymbol{v}_{p}=0_{2 \times 1}
$$

Hence, we have

$$
\left.I_{26} D_{L o i j} \frac{\partial \boldsymbol{\beta}_{o i j}}{\partial \varepsilon_{o i}^{T}}\right|_{0}=0_{2 \times 6}
$$

Two valid constraints are given from Eq. (47) and then $\boldsymbol{\theta}_{o j i}$ is eliminated. The grasp displacement parameters are reduced to

$$
\begin{gathered}
\boldsymbol{\alpha}^{o r}:=\left[\boldsymbol{\varepsilon}_{o i}^{T}, \boldsymbol{\theta}_{o i 1}^{T}, \phi_{o i 1}, \cdots, \boldsymbol{\theta}_{o i n_{j}}^{T}, \phi_{o i n_{j}}\right]^{T} \\
\in \mathbb{R}^{6+3 n_{j}}
\end{gathered}
$$

The superscript "or" is used for representing the rolling contact between the objects. The force vector and the grasp stiffness matrix are obtained by the following forms:

$$
\begin{gathered}
G^{o r}:=Z^{T} G^{o s} \in \mathbb{R}^{\left(6+3 n_{j}\right)} \\
H^{\text {or }}:=Z^{T} H^{o s} Z \in \mathbb{R}^{\left(6+3 n_{j}\right) \times\left(6+3 n_{j}\right)}
\end{gathered}
$$


where,

$$
\begin{gathered}
\boldsymbol{\alpha}^{o s}=Z \boldsymbol{\alpha}^{o r} \\
Z:=\text { blockdiag }\left[I_{6}, Z_{i 1}, \cdots, Z_{i n_{j}}\right] \in \mathbb{R}^{\left(6+5 n_{j}\right) \times\left(6+3 n_{j}\right)} \\
Z_{i j}:=\left[\begin{array}{cc}
0_{2 \times 1} \\
I_{2} \\
0_{1 \times 2} \\
{\left[I_{26}{ }^{\left.{ }^{o i j}{ }_{B_{L o j i}} C_{o j i}\right]^{-1}\left[I_{26} C_{o i j}\right]}\right.} & 0_{2 \times 1} \\
\in \mathbb{R}^{5 \times 3}
\end{array}\right.
\end{gathered}
$$

\section{Numerical Example}

In our analysis, the number of fingers is unlimited. Our analysis is applicable to various shapes because the contact surface geometry is included. If we show complicated examples, the reader cannot easily verify the numerical results obtained by our method and cannot intuitively judge the soundness of our method. So we show simple examples.

\subsection{Example 1}

In order to demonstrate the effectiveness of our method, we show the case that three objects are grasped by two fingers as shown in Fig. 3. The local contact frames and the parameters are set as the following values:

$$
\begin{aligned}
{ }^{o i} A_{\text {Loij }}:{ }^{o 0} A_{L o 01} & =\left[\begin{array}{cccc}
\boldsymbol{u}_{2} & \boldsymbol{u}_{3} & \boldsymbol{u}_{1} & l_{o} \boldsymbol{u}_{1} \\
& 0_{1 \times 3} & 1
\end{array}\right] \\
{ }^{o 0} A_{L o 02} & =A_{r z}(\pi){ }^{o 0} A_{L o 01}
\end{aligned}
$$

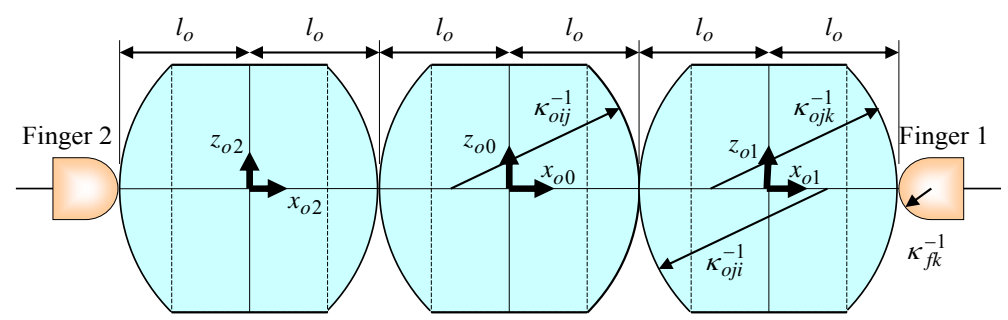

(a) Size of the objects and fingers
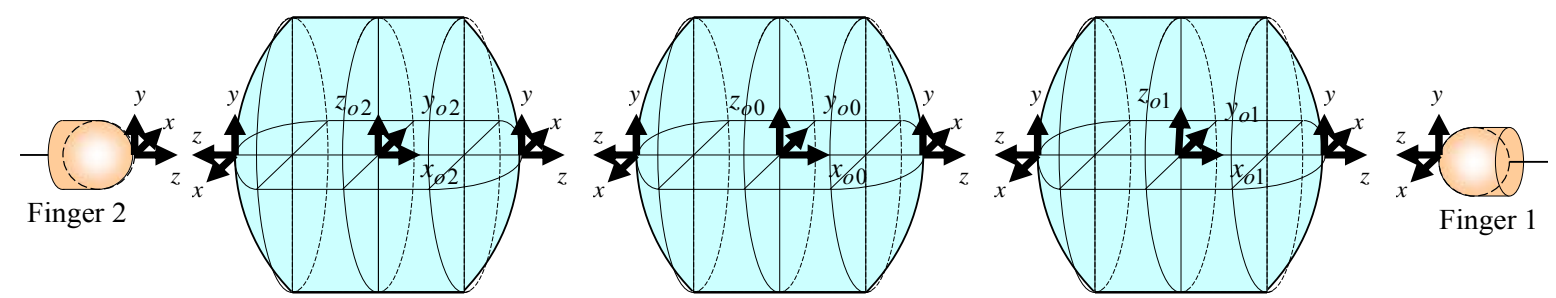

(b) Coordinate Frames

$$
\begin{gathered}
{ }^{o j} A_{\text {Loji }}:{ }^{o 1} A_{\text {Lo10 }}={ }^{o 0} A_{\text {Lo } 02}, \\
{ }^{o 2} A_{\text {Lo } 20}={ }^{o 0} A_{L o 01} \\
{ }^{o j} A_{\text {Lojk }}:{ }^{o 1} A_{L o 11}={ }^{o 0} A_{L o 01}, \\
{ }^{o 2} A_{L o 22}={ }^{o 0} A_{L o 02} \\
{ }^{f k} A_{L f k}:{ }^{f 1} A_{L f 1}={ }^{f 2} A_{L f 2}=A_{\text {cont }}
\end{gathered}
$$

$$
\phi_{o i j 0}=\pi, \phi_{f k 0}=\pi, S_{k}=s_{k} I_{3}, \boldsymbol{f}_{k}=f_{z} \boldsymbol{u}_{3}
$$

The matrices ${ }^{\text {Loij }} A_{\text {Coij }},{ }^{\text {Loji }} A_{\text {Coji }},{ }^{\text {Lojk }} A_{\text {Cojk }}$, and ${ }^{L f k} A_{C f k}$ are set as the following form:

$$
{ }^{L} A_{C}\left(\theta_{x}, \theta_{y}\right)=\left[\begin{array}{cc}
\cos \kappa \theta_{x} & -\sin \kappa \theta_{x} \sin \kappa \theta_{y} \\
0 & \cos \kappa \theta_{y} \\
-\sin \kappa \theta_{x} & -\cos \kappa \theta_{x} \sin \kappa \theta_{y} \\
0 & 0
\end{array}\right.
$$

$$
\left.\begin{array}{cc}
\sin \kappa \theta_{x} \cos \kappa \theta_{y} & \left(\sin \kappa \theta_{x} \cos \kappa \theta_{y}\right) / \kappa \\
\sin \kappa \theta_{y} & \left(\sin \kappa \theta_{y}\right) / \kappa \\
\cos \kappa \theta_{x} \cos \kappa \theta_{y} & \left(\cos \kappa \theta_{x} \cos \kappa \theta_{y}-1\right) / \kappa \\
0 & 1
\end{array}\right]
$$

The parameter $\kappa$ is a local curvature quantity. When the contact points are given at $\left(\theta_{x}, \theta_{y}\right)=$ $(0,0)$, the metric tensor $M$, curvature $K$, and torsion $T$ are given as the following values:

$$
\begin{gathered}
M_{o i j}=M_{o j i}=M_{o j k}=M_{f k}=I_{2}, \\
T_{o i j}=T_{o j i}=T_{o j k}=T_{f k}=\left[\begin{array}{ll}
0 & 0
\end{array}\right], \\
K_{o i j}=\kappa_{o i j} I_{2}, K_{o j i}=\kappa_{o j i} I_{2}, \\
K_{o j k}=\kappa_{o j k} I_{2}, K_{f k}=\kappa_{f k} I_{2} .
\end{gathered}
$$

Fig. 3 Three objects grasped by two fingers in a collinear line. 
If $\kappa$ is positive, zero, and negative, then the surface is convex, flat, and concave, respectively.

\subsubsection{Sliding Contact Case}

In the case of sliding contact, the grasp displacement parameters are given by

$$
\begin{aligned}
& \boldsymbol{\alpha}^{o S}=\left[\boldsymbol{\varepsilon}_{o 0}^{T}, \boldsymbol{\beta}_{01}^{T}, \boldsymbol{\beta}_{02}^{T}\right]^{T} \in \mathbb{R}^{16} \\
& h_{1,1}=2 s_{k}, \quad h_{2,2}=h_{3,3}=\frac{-2 \kappa_{o j k} \kappa_{f k} f_{z} s_{k}}{\left(\kappa_{o j k}+\kappa_{f k}\right) s_{k}^{\prime}}, \\
& h_{2,7}=h_{7,2}=-h_{2,12}=-h_{12,2}=h_{3,8}=h_{8,3}=h_{3,13}=h_{13,3}=\frac{f_{z} \kappa_{f k} s_{k}\left(\kappa_{o i j}-\kappa_{o j k}-2 l_{o} \kappa_{o i j} \kappa_{o j k}\right)}{\left(\kappa_{o j k}+\kappa_{f k}\right) s_{k}^{\prime}}, \\
& h_{2,10}=h_{10,2}=-h_{2,15}=-h_{15,2}=-h_{3,11}=-h_{11,3}=-h_{3,16}=-h_{16,3}=\frac{f_{z} \kappa_{f k} s_{k}\left(\kappa_{o j i}+\kappa_{o j k}-2 l_{o} \kappa_{o j i} \kappa_{o j k}\right)}{\left(\kappa_{o j k}+\kappa_{f k}\right) s_{k}^{\prime}} \\
& h_{5,5}=h_{6,6}=\frac{2 f_{z}\left(3 l_{o} \kappa_{o j k}-1\right)\left\{\kappa_{f k} f_{z}-\left(3 l_{o} \kappa_{f k}+1\right) s_{k}\right\}}{\left(\kappa_{o j k}+\kappa_{f k}\right) s_{k}^{\prime}}, \\
& h_{5,8}=h_{8,5}=-h_{5,13}=-h_{13,5}=-h_{6,7}=-h_{7,6}=-h_{6,12}=-h_{12,6} \\
& =f_{z}\left(\kappa_{o i j}-\kappa_{o j k}-2 l_{o} \kappa_{o i j} \kappa_{o j k}\right) \times \frac{\left\{f_{z} \kappa_{f k}-\left(3 l_{o} \kappa_{f k}+1\right) s_{k}\right\}}{\left(\kappa_{o j k}+\kappa_{f k}\right) s_{k}^{\prime}}, \\
& h_{5,11}=h_{11,5}=-h_{5,16}=-h_{16,5}=h_{6,10}=h_{10,6}=h_{6,15}=h_{15,6} \\
& =f_{z}\left(2 l_{o} \kappa_{o j i} \kappa_{o j k}-\kappa_{o j i}-\kappa_{o j k}\right) \times \frac{\left\{f_{z} \kappa_{f k}-\left(3 l_{o} \kappa_{f k}+1\right) s_{k}\right\}}{\left(\kappa_{o j k}+\kappa_{f k}\right) s_{k}^{\prime}}, \\
& h_{7,7}=h_{8,8}=h_{12,12}=h_{13,13}=f_{z}\left(2 l_{o} \kappa_{o i j} \kappa_{o j k}-\kappa_{o i j}+\kappa_{o j k}\right) \times \frac{\left\{f_{z} \kappa_{o i j} \kappa_{f k}-\left(2 l_{o} \kappa_{o i j} \kappa_{f k}+\kappa_{o i j}+\kappa_{f k}\right) s_{k}\right\}}{\left(\kappa_{o j k}+\kappa_{f k}\right) s_{k}^{\prime}}, \\
& h_{7,10}=h_{10,7}=-h_{8,11}=-h_{11,8}=h_{12,15}=h_{15,12}=-h_{13,16}=-h_{16,13} \\
& =f_{z}\left(2 l_{o} \kappa_{o j i} \kappa_{o j k}-\kappa_{o j i}-\kappa_{o j k}\right) \times \frac{\left\{f_{z} \kappa_{o i j} \kappa_{f k}-\left(2 l_{o} \kappa_{o i j} \kappa_{f k}+\kappa_{o i j}+\kappa_{f k}\right) s_{k}\right\}}{\left(\kappa_{o j k}+\kappa_{f k}\right) s_{k}^{\prime}}, \\
& h_{10,10}=h_{11,11}=h_{15,15}=h_{16,16}=f_{z}\left(2 l_{o} \kappa_{o j i} \kappa_{o j k}-\kappa_{o j i}-\kappa_{o j k}\right) \times \frac{\left\{f_{z} \kappa_{o j i} \kappa_{f k}-\left(2 l_{o} \kappa_{o j i} \kappa_{f k}+\kappa_{o j i}-\kappa_{f k}\right) s_{k}\right\}}{\left(\kappa_{o j k}+\kappa_{f k}\right) s_{k}^{\prime}} \text {. }
\end{aligned}
$$

The other elements are zero. The matrix $H^{o s}$ becomes a symmetric matrix. From Eq. (32), the stability condition of the fingers becomes

$$
\begin{gathered}
U_{j k, \gamma \gamma}^{r c}=8 s_{k}^{\prime} I_{2} \\
s_{k}^{\prime}:=s_{k}-f_{z} \frac{\kappa_{f k} \kappa_{o j k}}{\kappa_{f k}+\kappa_{o j k}}
\end{gathered}
$$

If the matrix $U_{k, \gamma \gamma}^{r c}$ is positive definite, the fingers are stable. Even if either $\kappa_{o j k}=0$ or $\kappa_{f k}=0$, the grasp stiffness matrix is obtained. It is shown that the proposed method does not fail in the calculation.

The length and the curvature quantities are set as the following values:

$$
l_{o}=\kappa_{o i j}^{-1}=\kappa_{o j i}^{-1}=\kappa_{o j k}^{-1}=0.02[\mathrm{~m}],
$$

$$
\begin{gathered}
\kappa_{f k}^{-1}=0.005[\mathrm{~m}], s_{k}=500[\mathrm{~N} / \mathrm{m}], \\
f_{z}=2.5[\mathrm{~N}] .
\end{gathered}
$$

These settings satisfy the following conditions:

$$
s_{k}^{\prime}>0, \kappa_{o j k}+\kappa_{f k}>0, \kappa_{o i j}+\kappa_{o j i}>0 \text {. }
$$

From the above conditions, the matrix $U_{k, \gamma \gamma}^{r c}$ is positive definite and (A2) is satisfied. Table 1 shows the eigenvalues and the eigenvectors of Eq. (55) in descending order. The grasp displacements are decomposed into 16 modes, where each element of the eigenvectors corresponds to the parameter Eq. (54). Some of these modes are shown in Fig. 4. The dashed lines mean the initial grasp, and the solid lines mean the displaced grasp. The displacement of Mode 1 is 
Table 1 Eigenvalues and eigenvectors of sliding contact case.

\begin{tabular}{|c|c|c|c|c|c|c|c|c|c|}
\hline \multirow{3}{*}{ Mode } & \multirow{3}{*}{ Eigenvalue } & \multicolumn{8}{|c|}{ Eigenvector } \\
\hline & & \multicolumn{2}{|l|}{$\overline{\varepsilon_{o 0}^{T}}$} & \multicolumn{3}{|l|}{$\boldsymbol{\beta}_{01}^{T}$} & \multicolumn{3}{|l|}{$\overline{\boldsymbol{\beta}_{02}^{T}}$} \\
\hline & & $\boldsymbol{x}_{o 0}^{T}$ & $\xi_{o 0}^{T}$ & $\boldsymbol{\theta}_{001}^{T}$ & $\phi_{o 01}$ & $\boldsymbol{\theta}_{o 10}^{T}$ & $\boldsymbol{\theta}_{002}^{T}$ & $\phi_{o 02}$ & $\boldsymbol{\theta}_{o 20}^{T}$ \\
\hline 1 & 1,000 & $1,0,0$ & $0,0,0$ & 0,0 & 0 & 0,0 & 0,0 & 0 & 0,0 \\
\hline 2 & 0 & $0,0,0$ & $1,0,0$ & 0,0 & 0 & 0,0 & 0,0 & 0 & 0,0 \\
\hline 3 & 0 & $0,0,0$ & $0,1,0$ & 0,1 & 0 & 0,0 & $0,-1$ & 0 & 0,0 \\
\hline 4 & 0 & $0,0,0$ & $0,0,1$ & $-1,0$ & 0 & 0,0 & $-1,0$ & 0 & 0,0 \\
\hline 5 & 0 & $0,0,0$ & $0,0,0$ & 0,0 & 1 & 0,0 & 0,0 & 0 & 0,0 \\
\hline 6 & 0 & $0,0,0$ & $0,0,0$ & 0,0 & 0 & 1,0 & 0,0 & 0 & 0,0 \\
\hline 7 & 0 & $0,0,0$ & $0,0,0$ & 0,0 & 0 & 0,1 & 0,0 & 0 & 0,0 \\
\hline 8 & 0 & $0,0,0$ & $0,0,0$ & 0,0 & 0 & 0,0 & 0,0 & 1 & 0,0 \\
\hline 9 & 0 & $0,0,0$ & $0,0,0$ & 0,0 & 0 & 0,0 & 0,0 & 0 & 1,0 \\
\hline 10 & 0 & $0,0,0$ & $0,0,0$ & 0,0 & 0 & 0,0 & 0,0 & 0 & 0,1 \\
\hline 11 & -66.99 & $0,1,0$ & $0,0,0$ & $-0.366,0$ & 0 & 0,0 & $0.366,0$ & 0 & 0,0 \\
\hline 12 & -66.99 & $0,0,1$ & $0,0,0$ & $0,-0.366$ & 0 & 0,0 & $0,-0.366$ & 0 & 0,0 \\
\hline 13 & -750.6 & $0,0,0$ & $0,0.04,0$ & $0,-1$ & 0 & 0,0 & 0,1 & 0 & 0,0 \\
\hline 14 & -750.6 & $0,0,0$ & $0,0,0.04$ & 1,0 & 0 & 0,0 & 1,0 & 0 & 0,0 \\
\hline 15 & -933.0 & $0,0.732,0$ & $0,0,0$ & 1,0 & 0 & 0,0 & $-1,0$ & 0 & 0,0 \\
\hline 16 & -933.0 & $0,0,0.732$ & $0,0,0$ & 0,1 & 0 & 0,0 & 0,1 & 0 & 0,0 \\
\hline
\end{tabular}

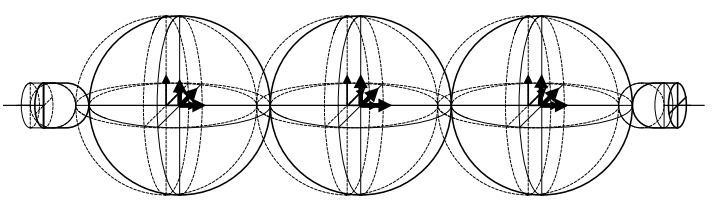

(a) Mode 1

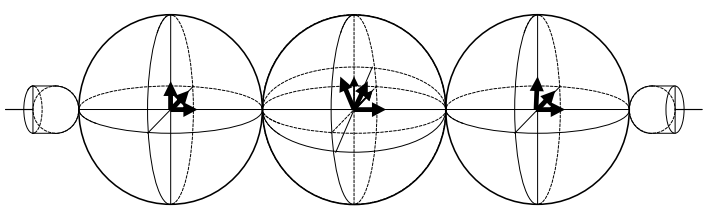

(b) Mode 2

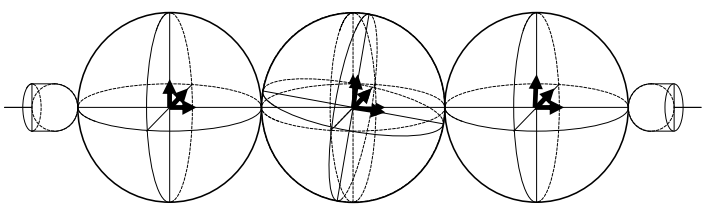

(c) Mode 3

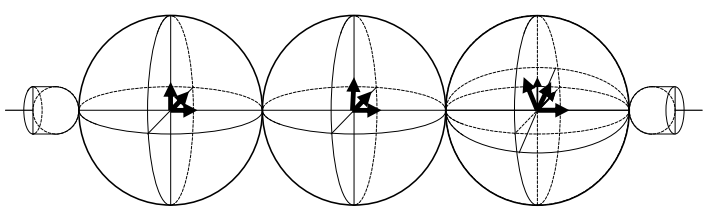

(d) Mode 5

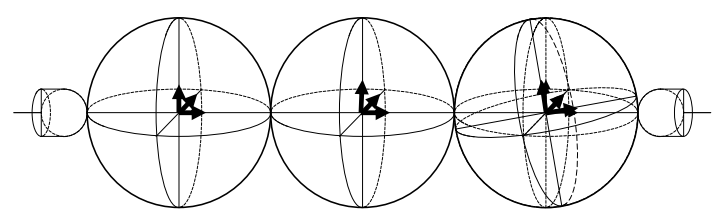

(e) Mode 7

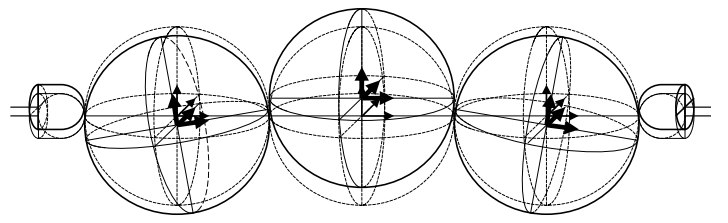

(f) Mode 12

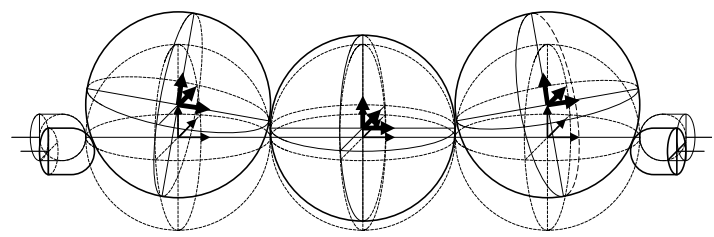

(g) Mode 16

Fig. 4 Modes of the grasp with sliding contact. 
shown in Fig. 4a. In this mode, the three objects move in the $x_{o 0}$ axis. Because the restoring force is generated from the fingers, this mode is stable (the eigenvalue is positive). Mode 2 is shown in Fig. $4 \mathrm{~b}$. Because, in this mode, the central object is rotated solely, the finger position does not change, the potential energy does not change, and this mode is critical (the eigenvalue is zero). Fig. 4c-4e shows Mode 3, 5, 7, respectively. In these modes, one of the objects is rotated solely. Fig. $4 \mathrm{f}-4 \mathrm{~g}$ shows Mode 12 and 16, respectively. In these modes, each object slides and is pushed out by the finger forces. Hence, these modes are unstable (the eigenvalues are negative). This grasp is unstable from Modes 11-16. In practical case, the grasp displacement gets into the unstable modes due to an external disturbance, the grasp parameters error, the objects' shape error, and so on. The stable modes are concealed by the unstable modes and human beings experience the unstable modes only. It is thought that human beings learn these properties in their own daily life and then intuitively judge the grasp unstable.

\subsubsection{Rolling Contact Case}

In the case of rolling contact, the independent parameters of the grasp displacement are given by

$\boldsymbol{\alpha}^{o r}:=\left[\boldsymbol{\varepsilon}_{o 0}^{T}, \boldsymbol{\theta}_{o 01}^{T}, \phi_{o 01}, \boldsymbol{\theta}_{o 02}^{T}, \phi_{o 02}\right]^{T} \in \mathbb{R}^{12}$.

The grasp stiffness matrix with rolling contact is given by

$$
H^{\text {or }}=\left\{h_{p, q}\right\} \in \mathbb{R}^{12 \times 12},
$$

where

$$
\begin{gathered}
h_{1,1}=h_{2,2}=h_{3,3}=2 s_{k}, \\
h_{2,7}=h_{7,2}=-h_{2,10}=-h_{10,2}=h_{3,8}=h_{8,3} \\
=h_{3,11}=h_{11,3}=2\left(\kappa_{o i j}+\kappa_{o j i}\right) l_{o} s_{k}, \\
h_{5,5}=h_{6,6}=2\left\{3\left(3 l_{o} s_{k}-f_{z}\right) l_{o}+\frac{f_{z}}{\kappa_{o j k}+\kappa_{f k}}\right\}, \\
-h_{5,8}=-h_{8,5}=h_{5,11}=h_{11,5} \\
=h_{6,7}=h_{7,6}=h_{6,10}=h_{10,6} \\
=\left(\kappa_{o i j}+\kappa_{o j i}\right) \times\left\{2\left(3 l_{o} s_{k}-f_{z}\right) l_{o}+\frac{f_{z}}{\kappa_{o j k}+\kappa_{f k}}\right\}, \\
h_{7,7}=h_{8,8}=h_{10,10}=h_{11,11} \\
=2 l_{o}\left(2 l_{o} s_{k}-f_{z}\right)\left(\kappa_{o i j}+\kappa_{o j i}\right)^{2}+ \\
\frac{f_{z}\left(\kappa_{o i j}+\kappa_{o j i}+\kappa_{o j k}+\kappa_{f k}\right)}{\kappa_{o j k}+\kappa_{f k}}\left(\kappa_{o i j}+\kappa_{o j i}\right) .
\end{gathered}
$$

The other elements are zero and $H^{\text {or }}$ becomes a

\begin{tabular}{|c|c|c|c|c|c|c|c|}
\hline \multirow{3}{*}{ Mode } & \multirow{3}{*}{ Eigenvalue } & \multicolumn{6}{|c|}{ Eigenvalue } \\
\hline & & \multicolumn{6}{|l|}{$\begin{array}{ll}\varepsilon_{o 0}^{T} \\
\end{array}$} \\
\hline & & $\boldsymbol{x}_{o 0}^{T}$ & $\xi_{o 0}^{T}$ & $\boldsymbol{\theta}_{o 01}^{T}$ & $\phi_{o 01}$ & $\boldsymbol{\theta}_{O 02}^{T}$ & $\phi_{002}$ \\
\hline 1 & $8,427.1$ & $0,0.539,0$ & $0,0,0$ & 1,0 & 0 & $-1,0$ & 0 \\
\hline 2 & $8,427.1$ & $0,0,0.539$ & $0,0,0$ & 0,1 & 0 & 0,1 & 0 \\
\hline 3 & $7,353.4$ & $0,0,0$ & $0,0.030,0$ & $0,-1$ & 0 & 0,1 & 0 \\
\hline 4 & $7,353.4$ & $0,0,0$ & $0,0,0.030$ & 1,0 & 0 & 1,0 & 0 \\
\hline 5 & 1,000 & $1,0,0$ & $0,0,0$ & 0,0 & 0 & 0,0 & 0 \\
\hline 6 & 0 & $0,0,0$ & $1,0,0$ & 0,0 & 0 & 0,0 & 0 \\
\hline 7 & 0 & $0,0,0$ & $0,0,0$ & 0,0 & 1 & 0,0 & 0 \\
\hline 8 & 0 & $0,0,0$ & $0,0,0$ & 0,0 & 0 & 0,0 & 1 \\
\hline 9 & -0.033 & $0,0,0$ & $0,0,1$ & $-0.015,0$ & 0 & $-0.015,0$ & 0 \\
\hline 10 & -0.033 & $0,0,0$ & $0,1,0$ & $0,0.015$ & 0 & $0,-0.015$ & 0 \\
\hline 11 & -77.13 & $0,1,0$ & $0,0,0$ & $-0.270,0$ & 0 & $0.270,0$ & 0 \\
\hline 12 & -77.13 & $0,0,1$ & $0,0,0$ & $0,-0.270$ & 0 & $0,-0.270$ & 0 \\
\hline
\end{tabular}
symmetric matrix. The eigenvalues and the eigenvectors of $H^{\text {or }}$ with the values Eq. (58) are shown in Table 2. In this case, the grasp displacements are decomposed into 12 modes. Five modes are stable, three modes are critical, and four modes are unstable. This grasp is unstable because the unstable modes exist. Fig. 5 shows some of these modes. In Mode 9-12, the grasps buckle due to the finger forces. These results are coincided with human intuition.

Table 2 Eigenvalues and eigenvectors of sliding contact case. 


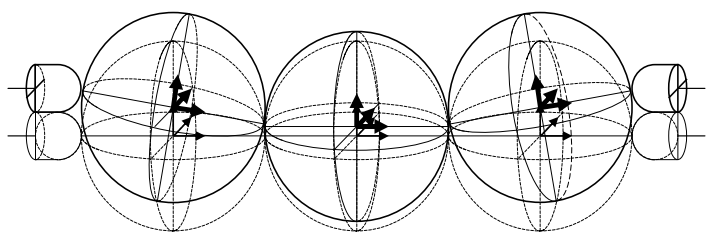

(a) Mode 2

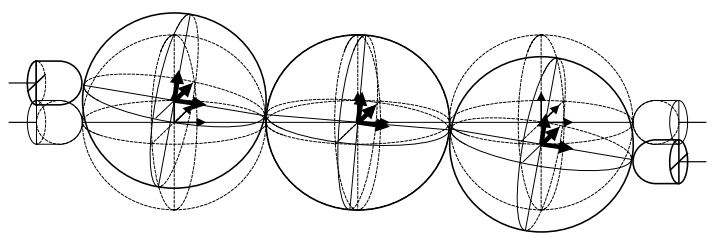

(b) Mode 4

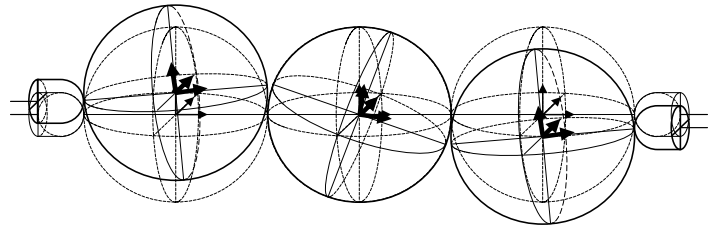

(c) Mode 10

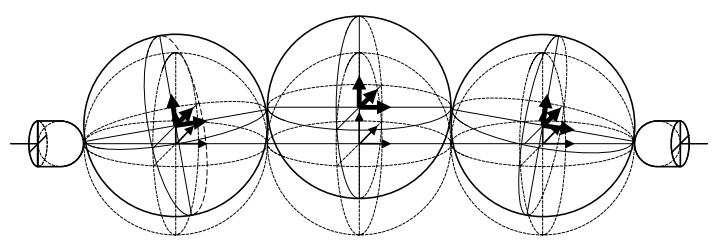

(d) Mode 12

Fig. 5 Grasp of three objects.

\subsection{Example 2}

We investigate the case that three objects are grasped by four fingers as shown in Fig. 6. The matrices ${ }^{o i} A_{\text {Loij }}$ and ${ }^{o j} A_{\text {Loji }}$ are set as the same values of Example 1. The local contact frames are set as the following values:

$$
\begin{gathered}
{ }^{o j} A_{L o j k}:{ }^{o 1} A_{L o 11}=A_{r z}(-\pi / 3){ }^{o 0} A_{L o 01}, \\
{ }^{o 1} A_{L o 12}=A_{r z}(\pi / 3){ }^{o 0} A_{L o 01}, \\
{ }^{o 2} A_{L o 23}=A_{r z}(2 \pi / 3){ }^{o 0} A_{L o 01}, \\
{ }^{o 2} A_{L o 24}=A_{r z}(-2 \pi / 3){ }^{o 0} A_{L o 01}, \\
\phi_{o i j 0}: \phi_{o 010}=\pi, \quad \phi_{o 020}=\pi / 2, \\
\kappa_{o i j}^{-1}=-0.04[\mathrm{~m}], \quad \kappa_{o j i}^{-1}=l_{o}=0.02[\mathrm{~m}], \\
\kappa_{o j k}^{-1}=-0.02[\mathrm{~m}], \kappa_{f k}^{-1}=0.005[\mathrm{~m}] .
\end{gathered}
$$

The stiffness $S_{k}$, the contact force $\boldsymbol{f}_{k}$, and the angle $\phi_{f k 0}$ are set as the same values shown in Example 1. The stiffness matrices $H^{o s}$ and $H^{o r}$ are omitted because those elements are somewhat complicated and long.

5.2.1 Grasp Stability versus the Object Surface Curvature $\boldsymbol{\kappa}_{\boldsymbol{o j} \boldsymbol{k}}$

Figs. 7 and 8 show the eigenvalues versus the object surface curvature $\kappa_{o j k}$, while the other parameters are fixed as shown in Eq. (63). In Figs. 7(b) and $8(\mathrm{~b})$, the vertical axis is magnified from (a). In the figures, the parameter $\kappa_{o j k}$ is given as the range from $-200\left(=-\kappa_{f k}\right)[1 / \mathrm{m}]$ to $100\left(=2 \kappa_{o j i}\right)[1 / \mathrm{m}]$. In this range, the value $s_{k}^{\prime}$ is positive and then $U_{j k, \gamma \gamma}^{r c}$ is positive definite. Tables 3 and 4 show the eigenvalues and eigenvectors when the parameters are given as Eq. (63). Fig. 7 shows the sliding contact case at all contact points. The outer objects are spherical objects when $\kappa_{o j k}=50[1 / \mathrm{m}]$. As shown in Table 3, the numbers of stable modes, critical modes, and unstable modes are 13, 1, and 2, respectively, when $\kappa_{o j k}=$ $-50[1 / \mathrm{m}]$. When the parameter $\kappa_{o j k}$ is larger than 50 $[1 / \mathrm{m}]$, the number of unstable modes increases because the number of negative eigenvalues increases. Fig. 8 shows the case with rolling contact. This grasp is critical in one direction. The direction is given in the $x_{o 0}$ axis rotation of the central object as shown in Table 4. In the other directions, the grasp is stable. The critical mode will be stabilized if a twist spring model along the contact normal is added.

From Figs. 7 and 8, it is shown that the grasp stability decreases when $\kappa_{o j k}$ increases.

5.2.2 Grasp Stability versus the Finger Surface Curvature $\boldsymbol{\kappa}_{\boldsymbol{f} \boldsymbol{k}}$

Figs. 9 and 10 show the eigenvalues versus the curvature $\kappa_{f k}$, while the other parameters are fixed as shown in Eq. (63). In the figures, the finger surface curvature $\kappa_{f k}$ is given as the range from $50\left(=-\kappa_{o j k}\right)$ $[1 / \mathrm{m}]$ to $200[1 / \mathrm{m}]$. Fig. 9 shows the sliding contact case at all contact points. Fig. 10 shows the rolling 


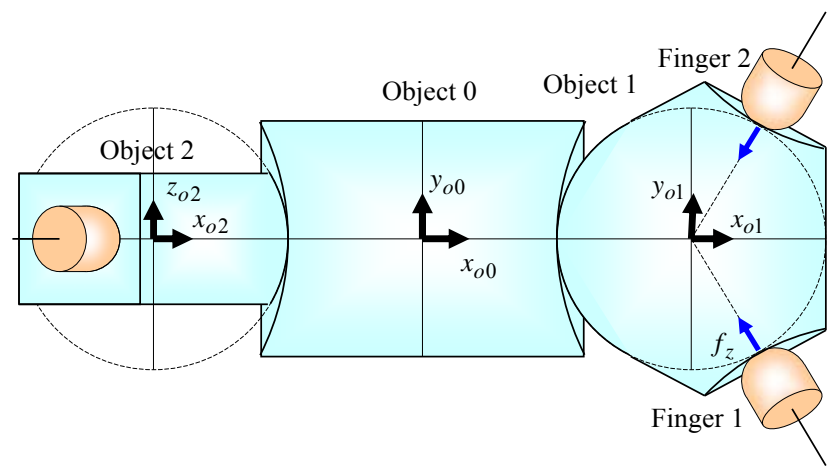

(a) Top view

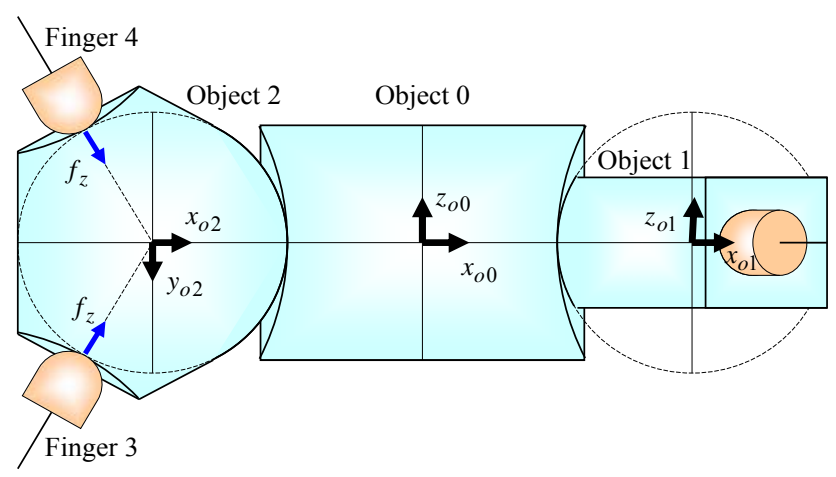

(b) Front view

Fig. 6 Three objects grasped by four fingers.

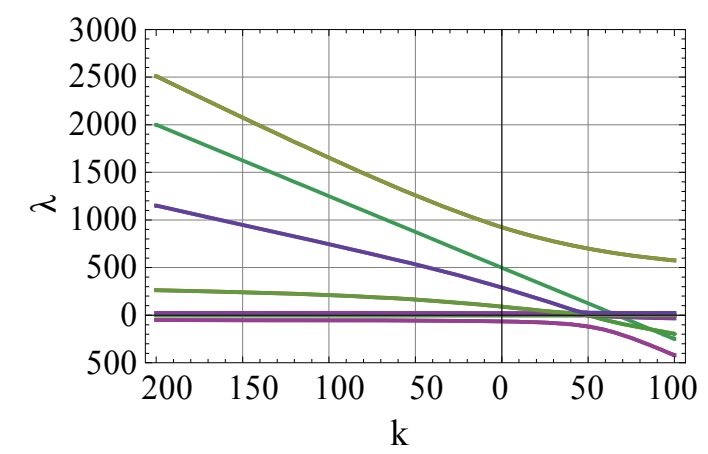

(a) Eigenvalues

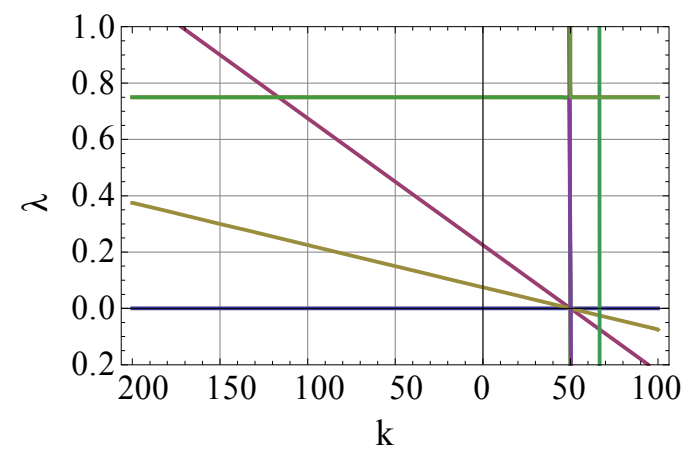

(b) Vertical axis magnified from (a)

Fig. 7 Eigenvalues of the grasp versus local curvature $\kappa_{o j k}$ with sliding contact.

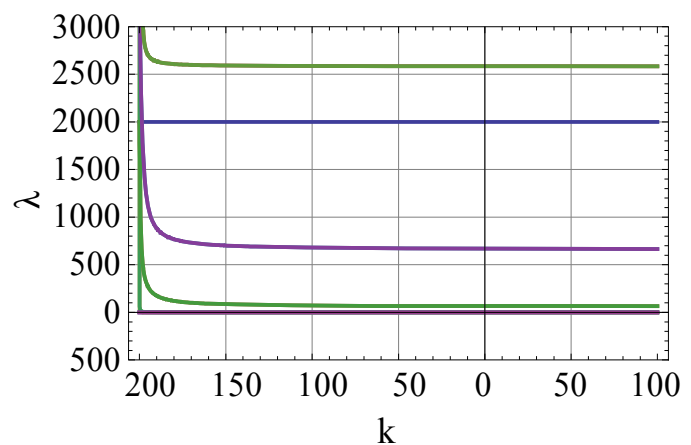

(a) Eigenvalues

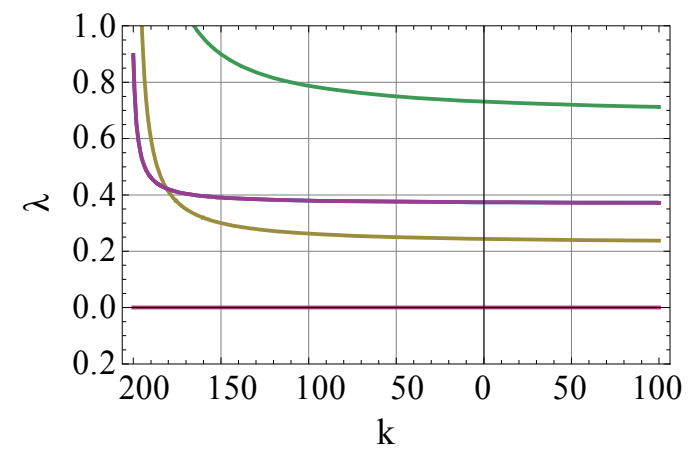

(b) Vertical axis magnified from (a)

Fig. 8 Eigenvalues of the grasp versus local curvature $\kappa_{o j k}$ with rolling contact. 
Table 3 Eigenvalues and eigenvectors of sliding contact case $\left(\kappa_{o j k}=-50, \kappa_{f k}=200\right)$.

\begin{tabular}{llllllllll}
\hline \multirow{2}{*}{ Mode } & \multirow{2}{*}{ Eigenvalue } & \multicolumn{7}{c}{ Eigenvector } \\
\cline { 3 - 9 } & & $\boldsymbol{\varepsilon}_{o 0}^{T}$ & $\boldsymbol{\beta}_{o 0}^{T}$ & $\boldsymbol{\beta}_{001}^{T}$ & $\phi_{o 01}$ & $\boldsymbol{\theta}_{o 10}^{T}$ & $\boldsymbol{\theta}_{002}^{T}$ & $\phi_{o 02}$ & $\boldsymbol{\theta}_{o 20}^{T}$ \\
\hline 1 & 1258 & $0,1,0$ & $0,0,0.022$ & $0.239,0$ & 0 & $0.291,0$ & $-0.011,0$ & 0 & $0,-0.221$ \\
2 & 1258 & $0,0,1$ & $0,0.022,0$ & $0,0.011$ & 0 & $0,-0.221$ & $0,0.240$ & 0 & $0.291,0$ \\
3 & 875 & $1,0,0$ & $0,0,0$ & 0,0 & 0 & 0,0 & 0,0 & 0 & 0,0 \\
4 & 534 & $0,-0.140,0$ & $0,0,0.026$ & $-0.548,0$ & 0 & 1,0 & $0.010,0$ & 0 & $0,0.088$ \\
5 & 534 & $0,0,-0.140$ & $0,0.026,0$ & $0,-0.010$ & 0 & $0,0.088$ & $0,-0.548$ & 0 & 1,0 \\
6 & 164 & $0,-0.054,0$ & $0,0,0.145$ & $0.768,0$ & 0 & $0.318,0$ & $0.381,0$ & 0 & 0,1 \\
7 & 164 & $0,0,-0.054$ & $0,0.145,0$ & $0,-0.381$ & 0 & 0,1 & $0,0.768$ & 0 & $0.318,0$ \\
8 & 23.4 & $0,0.401,0$ & $0,0,-0.020$ & $-0.780,0$ & 0 & $-0.414,0$ & 1,0 & 0 & $0,0.375$ \\
9 & 23.4 & $0,0,-0.401$ & $0,0.020,0$ & 0,1 & 0 & $0,-0.375$ & $0,0.780$ & 0 & $0.414,0$ \\
10 & 0.750 & $0,0.024,0$ & $0,0,1$ & $-0.103,0$ & 0 & $-0.078,0$ & $-0.101,0$ & 0 & $0,-0.001$ \\
11 & 0.750 & $0,0,0.024$ & $0,1,0$ & $0,0.101$ & 0 & $0,-0.001$ & $0,-0.103$ & 0 & $-0.078,0$ \\
12 & 0.45 & $0,0,0$ & $1,0,0$ & 0,0 & 0.5 & 0,0 & 0,0 & -0.5 & 0,0 \\
13 & 0.15 & $0,0,0$ & $0,0,0$ & 0,0 & 1 & 0,0 & 0,0 & 1 & 0,0 \\
14 & 0 & $0,0,0$ & $1,0,0$ & 0,0 & -1 & 0,0 & 0,0 & 1 & 0,0 \\
15 & -56.1 & $0,-0.392,0$ & $0,0,0.182$ & $0.495,0$ & 0 & $0.281,0$ & 1,0 & 0 & $0,-0.898$ \\
16 & -56.1 & $0,0,0.392$ & $0,-0.182,0$ & 0,1 & 0 & $0,0.898$ & $0,-0.495$ & 0 \\
\hline
\end{tabular}

Table 4 Eigenvalues and eigenvectors of rolling contact case $\left(\kappa_{o j k}=-50, \kappa_{f k}=200\right)$.

\begin{tabular}{|c|c|c|c|c|c|c|c|}
\hline \multirow{3}{*}{ Mode } & \multirow{3}{*}{ Eigenvalue } & \multicolumn{6}{|c|}{ Eigenvector } \\
\hline & & \multicolumn{2}{|l|}{$\begin{array}{l}\varepsilon_{o 0}^{T} \\
\end{array}$} & \multicolumn{2}{|l|}{$\boldsymbol{\beta}_{01}^{T}$} & \multicolumn{2}{|l|}{$\boldsymbol{\beta}_{02}^{T}$} \\
\hline & & $\overline{\boldsymbol{x}_{o 0}^{T}}$ & $\xi_{o 0}^{T}$ & $\boldsymbol{\theta}_{o 01}^{T}$ & $\phi_{o 01}$ & $\begin{array}{l}\boldsymbol{\theta}_{O 02}^{T} \\
T\end{array}$ & $\phi_{002}$ \\
\hline 1 & 2,586 & $0,0,1$ & $0,0.001,0$ & $0,0.375$ & 0 & $0,0.406$ & 0 \\
\hline 2 & 2,586 & $0,1,0$ & $0,0,0.001$ & $0.406,0$ & 0 & $-0.375,0$ & 0 \\
\hline 3 & 2,000 & $1,0,0$ & $0,0,0$ & 0,0 & 0 & 0,0 & 0 \\
\hline 4 & 673 & $0,0,-0.095$ & $0,0.108,0$ & $0,-0.833$ & 0 & 0,1 & 0 \\
\hline 5 & 673 & $0,-0.095,0$ & $0,0,0.108$ & 1,0 & 0 & $0.833,0$ & 0 \\
\hline 6 & 68.8 & $0,0,-0.689$ & $0,-0.052,0$ & 0,1 & 0 & $0,0.773$ & 0 \\
\hline 7 & 68.8 & $0,0.689,0$ & $0,0,0.0518$ & $-0.773,0$ & 0 & 1,0 & 0 \\
\hline 8 & 0.75 & $0,0,0$ & $1,0,0$ & 0,0 & 0.5 & 0,0 & -0.5 \\
\hline 9 & 0.376 & $0,0,-0.012$ & $0,1,0$ & $0,0.077$ & 0 & $0,-0.044$ & 0 \\
\hline 10 & 0.376 & $0,-0.012,0$ & $0,0,1$ & $-0.044,0$ & 0 & $-0.077,0$ & 0 \\
\hline 11 & 0.25 & $0,0,0$ & $0,0,0$ & 0,0 & 1 & 0,0 & 1 \\
\hline 12 & 0 & $0,0,0$ & $1,0,0$ & 0,0 & -1 & 0,0 & 1 \\
\hline
\end{tabular}

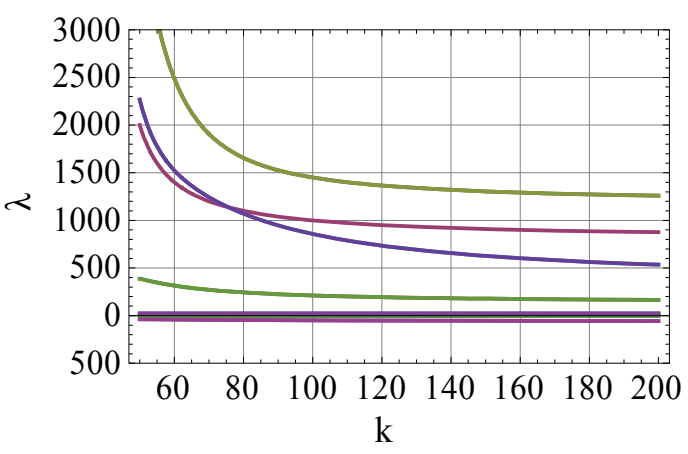

(a) Eigenvalues versus the curvature $\kappa_{f k}$

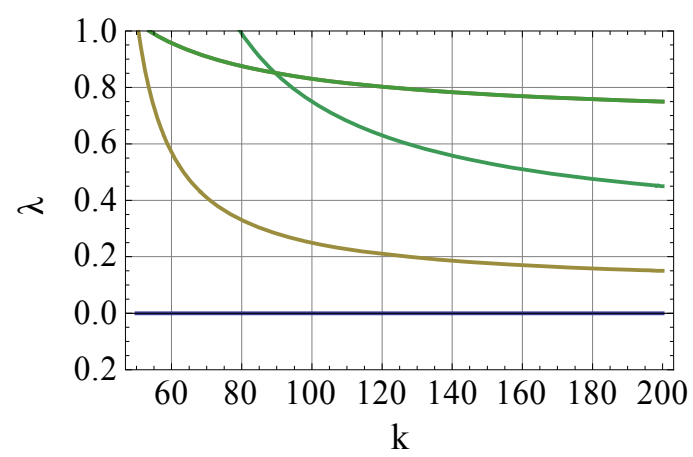

(b) Vertical axis magnified from (a)

Fig. 9 Eigenvalues of the grasp versus local curvature $\kappa_{f k}$ with sliding contact. 


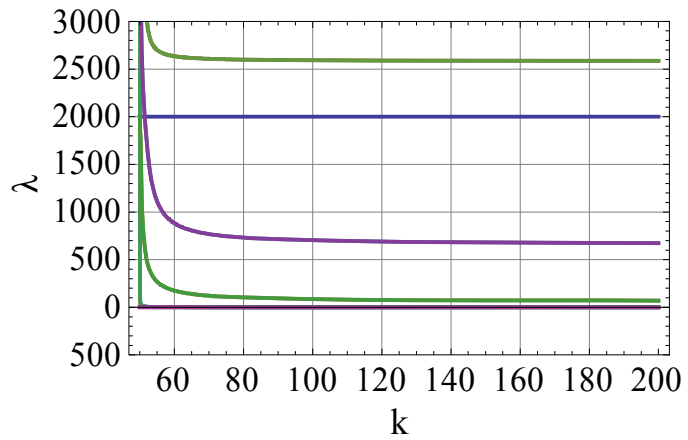

(a) Eigenvalues versus the curvature $\kappa_{f k}$

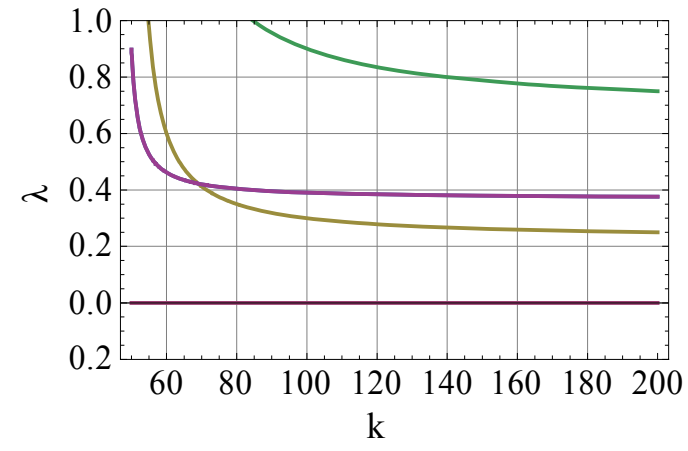

(b) Vertical axis magnified from (a)

Fig. 10 Eigenvalues of the grasp versus local curvature $\kappa_{f k}$ with rolling contact.

contact case. From Figs. 9 and 10, it is shown that the grasp stability decreases when $\kappa_{f k}$ increases.

\section{Conclusions}

The grasp stability in three dimensions was analyzed. The significant features of this paper are emphasized as follows: (i) The grasping of multiple objects was analyzed. (ii) The contact surface geometry (metric tensor, curvature and torsion) was included. (iii) Both the sliding contact and the rolling contact were investigated. In the case of rolling contact finger, the first- and the second-order partial derivatives of the contact position displacement parameters were derived. In the case of sliding contact, the novel independent parameters representing the contact position displacements were introduced and their partial derivatives were derived. (iv) The grasp force and moment vectors and the grasp stiffness matrices were analytically derived. The obtained formulas are clearer than those of the previous works. Moreover, the problems on surface curvatures were overcome. (v) The grasp stability was evaluated by using the eigenvalues of the matrix. The displacement direction of the grasp was obtained from its corresponding eigenvectors.

Because the surface geometry was considered, our analysis is applicable to various shaped spatial objects even if either the fingers or the objects are flat surfaces. Because not only rolling contact but also sliding contact was treated, our analysis is applicable to the cases such that the surface friction condition is low or unknown before grasping the objects.

Our method is applicable to grasp position planning for robots and fixture position planning for manufacturing systems. In our projected work, our proposed method is programmed and embedded to robots and automation systems, and then the planning is autonomously executed.

\section{Acknowledgment}

This work was supported in part by a Grant-in-Aid for Scientific Research (c), Japan, No. 23560285.

\section{References}

[1] Hanafusa, H., and Asada, H. 1982. "Stable Prehension by a Robot Hand with Elastic Fingers." In Robot Motion, edited by Brady, M. et al., 323-35. Massachusetts: MIT Press.

[2] Nguyen, V. D. 1988. "Constructing Stable Grasps." International Journal of Robotics Research 7(3): 3-16.

[3] Howard, W., and Kumar, V. 1996. "On the Stability of Grasped Objects." IEEE Transactions on Robotics and Automation 12 (6): 904-17.

[4] Rimon, E., and Burdick, J. 1998. "Mobility of Bodies in Contact. Part II: How Forces Are Generated by Curvature Effects." IEEE Transactions on Robotics and Automation 14 (5): 696-708.

[5] Yamada, T., Saha, S. K., Mimura, N., and Funahashi, Y. 1999. "Stability Analysis of Planar Grasp with 2D-Virtual Spring Model." Journal of Robotics and Mechatronics 11 (4): 274-82.

[6] Yamada, T., Taki, T., Yamada, M., Funahashi, Y., and Yamamoto, H. 2011. "Static Stability Analysis of Spatial Grasps Including Contact Surface Geometry." Advanced Robotics 25 (3): 447-72.

[7] Harada, K., Kaneko, M., Furutera, K., and Tsuji, T. 1999. 
"Active Force Closure for Multiple Objects." Journal of Robotics Society of Japan 17 (8): 1158-66.

[8] Harada, K., Nakano, S., Kaneko, M., and Tsuji, T. 2000. "Manipulation for Multiple Objects." Journal of Robotics Society of Japan 18 (2): 236-43.

[9] Yang, Y., Fukuda, K., and Tsujio, S. 2001. "On Computation of Grasp Internal Forces for Stably Grasping Multiple Objects." In Proceedings of the IEEE/RSJ International Conference on Intelligent Robots and Systems, 1776-81.

[10] Yoshikawa, T., Watanabe, T., and Daito, M. 2001. "Optimization of Power Grasps for Multiple Objects." In Proceedings of the IEEE International Conference on Robotics and Automation, 1786-91.

[11] Yamada, T., Yamanaka, S., Yamada, M., Funahashi, Y., and Yamamoto, H. 2010. "Static Stability Analysis of Grasping Multiple Objects in 2D." International Journal of Information Acquisition 7 (2): 119-34.

[12] Yamada, T., Taki, T., Yamada, M., and Yamamoto, H. 2012. "Static Grasp Stability Analysis of Two Spatial Objects Including Contact Surface Geometry." Transactions on Control and Mechanical Systems 1 (5): 218-28.

[13] Yamada, T. 2013. "Grasp Stability Analysis of Multiple Objects including Contact Surface Geometry in 3D." In Proceedings of the IEEE International Conference on Mechatronics and Automation, 36-43.

[14] Murray, M., Li, Z., and Sastry, S. S. 1994. A Mathematical Introduction to Robotic Manipulation. Florida: CRC Press.

\section{Appendix A: Nomenclatures}

A homogeneous transformation matrix of $\Sigma_{b}$ with respect to $\Sigma_{a}$ is defined by the following form:

$$
{ }^{a} A_{b}:=\left[\begin{array}{cc}
{ }^{a} R_{b} & { }^{a} \boldsymbol{p}_{b} \\
0_{1 \times 3} & 1
\end{array}\right] \in \mathbb{R}^{4 \times 4},
$$

where ${ }^{a} \boldsymbol{p}_{b} \in \mathbb{R}^{3}$ and ${ }^{a} R_{b} \in \mathbb{R}^{3 \times 3}$ represent position and orientation components, respectively. We also use the following matrices:

$$
\begin{gathered}
{ }^{a} B_{b}:=\left[\begin{array}{cc}
{ }^{a} R_{b} & { }^{a} \boldsymbol{p}_{b} \otimes{ }^{a} R_{b} \\
0_{3 \times 3} & { }^{a} R_{b}
\end{array}\right] \in \mathbb{R}^{6 \times 6}, \quad{ }^{a} W_{b}:=\left[\begin{array}{c}
{ }^{a} R_{b} \\
{ }^{a} \boldsymbol{p}_{b} \otimes{ }^{a} R_{b}
\end{array}\right]=\left[I_{3 x}{ }^{b} B_{a}\right]^{T} \in \mathbb{R}^{6 \times 3}, \\
I_{3 x}:=\left[I_{3}, 0_{3 \times 3}\right], I_{3 \xi}:=\left[0_{3 \times 3}, I_{3}\right] .
\end{gathered}
$$

The matrices have the feature as ${ }^{a} A_{c}={ }^{a} A_{b}{ }^{b} A_{c}$ and ${ }^{a} B_{c}={ }^{a} B_{b}{ }^{b} B_{c}$. The symbol $\otimes$ is used for representing the cross product in three dimensions. A vector $\varepsilon=\left[\boldsymbol{x}^{T}, \xi^{T}\right]^{T} \in \mathbb{R}^{6}$ represents a pose (position and orientation) displacement, where $\boldsymbol{x}=[x, y, z]^{T} \in \mathbb{R}^{3}$ and $\xi=[\xi, \eta, \zeta]^{T} \in \mathbb{R}^{3}$ are translation and rotation components, respectively.

$$
\begin{aligned}
& { }^{b o j} A_{o j}\left(\varepsilon_{o j}\right):=A_{\text {trans }}\left(x_{o j}\right) A_{\text {rot }}\left(\xi_{o j}\right), \quad A_{\text {trans }}(\boldsymbol{x}):=\left[\begin{array}{cc}
I_{3} & \boldsymbol{x} \\
0_{1 \times 3} & 1
\end{array}\right], \quad A_{\text {rot }}(\xi):=\left[\begin{array}{cc}
\operatorname{Rot}(\xi) & 0_{3 \times 1} \\
0_{1 \times 3} & 1
\end{array}\right], \\
& { }^{C f k} A_{\text {Cojk }}\left(\phi_{f k}\right):=A_{r z}\left(\phi_{f k}\right) A_{r z}\left(\phi_{f k 0}\right) A_{\text {cont }}, \quad{ }^{C o i j} A_{\text {Coji }}\left(\phi_{o i j}\right):=A_{r z}\left(\phi_{o i j}\right) A_{r z}\left(\phi_{o i j 0}\right) A_{c o n t}, \\
& A_{r z}(\phi):=\left[\begin{array}{cc}
\mathrm{R}_{z}(\phi) & 0_{3 \times 1} \\
0_{1 \times 3} & 1
\end{array}\right], \quad \mathrm{R}_{z}(\phi):=\left[\begin{array}{ccc}
\cos \phi & -\sin \phi & 0_{2 \times 1} \\
\sin \phi & \cos \phi & 1 \\
0_{1 \times 2} & 1
\end{array}\right] \text {, } \\
& A_{\text {cont }}:=\operatorname{diag}[1,-1,-1,1], \quad I_{34}:=\left[I_{3}, 0_{3 \times 1}\right], \quad \boldsymbol{v}_{p}:=\left[\begin{array}{c}
0_{3 \times 1} \\
1
\end{array}\right], \quad \boldsymbol{u}_{r z}:=\left[\begin{array}{c}
0_{3 \times 1} \\
\boldsymbol{u}_{3}
\end{array}\right], \\
& \boldsymbol{u}_{1}:=[1,0,0]^{T}, \quad \boldsymbol{u}_{2}:=[0,1,0]^{T}, \quad \boldsymbol{u}_{3}:=[0,0,1]^{T}, \\
& I_{23}:=\left[I_{2}, 0_{2 \times 1}\right], I_{24}:=\left[I_{2}, 0_{2 \times 4}\right], I_{26}:=\left[I_{2}, 0_{2 \times 4}\right] \text {. }
\end{aligned}
$$

Let the symbol $I_{n}$ be an n-by-n identity matrix, $\phi_{f k 0}$ and $\phi_{o i j 0}$ be initial spin angles, and diag[-] be a diagonal matrix.

\section{Appendix B: Contact Surface Geometry}

A parameter $\boldsymbol{\theta} \in \mathbb{R}^{2}$ represents a contact location displacement. The pose of contact frame $\Sigma_{C}$ with respect to the local frame $\Sigma_{L}$ is represented by

$$
{ }^{L} A_{C}(\boldsymbol{\theta}):=\left[\begin{array}{cc}
{ }^{L} R_{C}(\boldsymbol{\theta}) & { }^{L} \boldsymbol{p}_{C}(\boldsymbol{\theta}) \\
0_{1 \times 3} & 1
\end{array}\right],{ }^{L} A_{C}(0)=I_{4} .
$$


The body velocity of $\Sigma_{C}$ in $\Sigma_{L}$ is calculated by

$$
\begin{gathered}
\widehat{V}_{L, C}^{b}(t):={ }^{L} A_{C}^{-1}(t){ }^{L} \dot{A}_{C}(t)=\left[\begin{array}{cccc}
0 & -T M \dot{\boldsymbol{\theta}} & K M \dot{\boldsymbol{\theta}} & M \dot{\boldsymbol{\theta}} \\
T M \dot{\boldsymbol{\theta}} & 0 & & 0 \\
-[K M \dot{\boldsymbol{\theta}}]^{T} & 0 & 0 \\
0_{1 \times 3} & & 0
\end{array}\right]=\left[\begin{array}{cc}
{\left[\left(I_{3 \xi} C \dot{\boldsymbol{\theta}}\right) \otimes\right]} & I_{3 x} C \dot{\boldsymbol{\theta}} \\
0_{1 \times 3} & 0
\end{array}\right], \\
V_{L, C}^{b}(t)=C \dot{\boldsymbol{\theta}}, C:=\left[\begin{array}{c}
I_{2} \\
0_{1 \times 2} \\
\Omega K \\
T
\end{array}\right] M \in \mathbb{R}^{6 \times 2}, \Omega:=\left[\begin{array}{cc}
0 & -1 \\
1 & 0
\end{array}\right],
\end{gathered}
$$

where $M \in \mathbb{R}^{2 \times 2}, K \in \mathbb{R}^{2 \times 2}$ and $T \in \mathbb{R}^{1 \times 2}$ are the metric tensor, curvature and torsion of the body surface, respectively [14]. The square matrix $M$ is nonsingular. This definition is used for the following matrices: ${ }^{\text {Loij }} A_{\text {Coij }},{ }^{\text {Loji }} A_{\text {Coji }},{ }^{\text {Lojk }} A_{\text {Cojk }},{ }^{L f k} A_{C f k}$.

\section{Appendix C: Partial Derivatives of (5)}

The first-order partial derivatives of the object displacement are given by

$$
\begin{gathered}
\frac{\partial^{b o i} A_{o i}\left(\boldsymbol{\varepsilon}_{o i}\right)}{\partial x_{o i}}=\left[\frac{\partial^{b o i} A_{o i}\left(\boldsymbol{\varepsilon}_{o i}\right)}{\partial x_{o i}}{ }^{b o i} A_{o i}^{-1}\left(\boldsymbol{\varepsilon}_{o i}\right)\right]{ }^{b o i} A_{o i}\left(\boldsymbol{\varepsilon}_{o i}\right)=\left[\begin{array}{cc}
0_{3 \times 3} & \boldsymbol{u}_{1} \\
0_{1 \times 3} & 0
\end{array}\right]{ }^{b o i} A_{o i}\left(\boldsymbol{\varepsilon}_{o i}\right), \\
\frac{\partial^{b o i} A_{o i}\left(\boldsymbol{\varepsilon}_{o i}\right)}{\partial \xi_{o i}}={ }^{b o i} A_{o i}\left(\boldsymbol{\varepsilon}_{o i}\right)\left[{ }^{b o i} A_{o i}^{-1}\left(\boldsymbol{\varepsilon}_{o i}\right) \frac{\partial^{b o i} A_{o i}\left(\boldsymbol{\varepsilon}_{o i}\right)}{\partial \xi_{o i}}\right]={ }^{b o i} A_{o i}\left(\boldsymbol{\varepsilon}_{o i}\right)\left[\begin{array}{cc}
\left.\boldsymbol{u}_{1} \otimes\right] & 0_{3 \times 1} \\
0_{1 \times 3} & 0
\end{array}\right] .
\end{gathered}
$$

The first-order partial derivative of the spin angle between two bodies is given by

$$
\frac{\partial^{C f k} A_{C o j k}\left(\phi_{f k}\right)}{\partial \phi_{f k}}=\left[\frac{{ }^{C f k} A_{C o j k}\left(\phi_{f k}\right)}{\partial \phi_{f k}}{ }^{C f k} A_{C o j k}^{-1}\left(\phi_{f k}\right)\right]^{C f k} A_{C o j k}\left(\phi_{f k}\right)=\left[\begin{array}{cc}
{\left[\boldsymbol{u}_{3} \otimes\right]} & 0_{3 \times 1} \\
0_{1 \times 3} & 0
\end{array}{ }^{C f k} A_{C o j k}\left(\phi_{f k}\right) .\right.
$$

The first-order partial derivative of the contact location is given by

$$
\frac{\partial^{L f k} A_{C f k}\left(\boldsymbol{\theta}_{f k}\right)}{\partial \theta_{x f k}}={ }^{L f k} A_{C f k}\left(\boldsymbol{\theta}_{f k}\right)\left[{ }^{L f k} A_{C f k}^{-1}\left(\boldsymbol{\theta}_{f k}\right) \frac{\partial^{L f k} A_{C f k}\left(\boldsymbol{\theta}_{f k}\right)}{\partial \theta_{x f k}}\right]={ }^{L f k} A_{C f k}\left(\boldsymbol{\theta}_{f k}\right)\left[\begin{array}{c}
\left.\left(I_{3 \xi} C_{f k}\left[\begin{array}{l}
1 \\
0
\end{array}\right]\right) \otimes\right] \\
0_{1 \times 3}
\end{array} \quad \begin{array}{c}
I_{3 x} C_{f k}\left[\begin{array}{l}
1 \\
0
\end{array}\right] \\
0
\end{array}\right],
$$

where, $\boldsymbol{\theta}_{f k}=\left[\theta_{x f k}, \theta_{y f k}\right]^{T}$.

In general, the first-order partial derivative of finger position displacement is given by the following form:

$$
{ }^{a} A_{b}\left[\begin{array}{cc}
{\left[\boldsymbol{\omega}_{b} \otimes\right]} & \boldsymbol{v}_{b} \\
0_{1 \times 3} & 0
\end{array}\right]{ }^{a} A_{b}^{-1}=\left[\begin{array}{cc}
{\left[\left({ }^{a} R_{b} \boldsymbol{\omega}_{b}\right) \otimes\right]} & I_{3 x}{ }^{a} B_{b}\left[\begin{array}{c}
\boldsymbol{v}_{b} \\
\boldsymbol{\omega}_{b}
\end{array}\right] \\
0_{1 \times 3} & 0
\end{array}\right]=\left[\begin{array}{cc}
\left(\begin{array}{cc}
\left.I_{3 \xi}{ }^{a} B_{b}\left[\begin{array}{c}
\boldsymbol{v}_{b} \\
\boldsymbol{\omega}_{b}
\end{array}\right]\right) \otimes \\
0_{1 \times 3}
\end{array}\right] I_{3 x}{ }^{a} B_{b}\left[\begin{array}{c}
\boldsymbol{v}_{b} \\
\boldsymbol{\omega}_{b}
\end{array}\right] \\
0
\end{array}\right] .
$$

The second-order partial derivative of the finger position displacement is given by the following form:

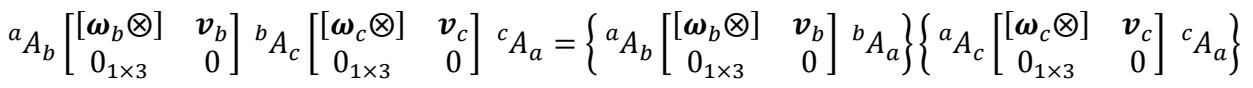

$$
\begin{aligned}
& =\left[\begin{array}{cc}
{\left[\left({ }^{a} R_{b} \boldsymbol{\omega}_{b}\right) \otimes\right]\left[\left({ }^{a} R_{c} \boldsymbol{\omega}_{c}\right) \otimes\right]} & {\left[\left({ }^{a} R_{b} \boldsymbol{\omega}_{b}\right) \otimes\right] I_{3 x}{ }^{a} B_{c}\left[\begin{array}{l}
\boldsymbol{v}_{c} \\
\boldsymbol{\omega}_{c}
\end{array}\right]} \\
0_{1 \times 3} & 0
\end{array}\right] .
\end{aligned}
$$

Hence we have

$$
\boldsymbol{f}_{k}^{T} I_{34}\left\{{ }^{a} A_{b}\left[\begin{array}{cc}
{\left[\boldsymbol{\omega}_{b} \otimes\right]} & \boldsymbol{v}_{b} \\
0_{1 \times 3} & 0
\end{array}\right]{ }^{b} A_{c}\left[\begin{array}{cc}
{\left[\boldsymbol{\omega}_{c} \otimes\right]} & \boldsymbol{v}_{c} \\
0_{1 \times 3} & 0
\end{array}\right]{ }^{c} A_{a}\right\} \boldsymbol{v}_{p}=-\left\{{ }^{a} B_{b}\left[\begin{array}{l}
\boldsymbol{v}_{b} \\
\boldsymbol{\omega}_{b}
\end{array}\right]\right\} F_{k}\left\{{ }^{a} B_{c}\left[\begin{array}{l}
\boldsymbol{v}_{c} \\
\boldsymbol{\omega}_{c}
\end{array}\right]\right\}
$$

where,

$$
F_{k}:=I_{3 \xi}^{T}\left[\boldsymbol{f}_{k} \otimes\right] I_{3 x}=\left[\begin{array}{cc}
0_{3 \times 3} & 0_{3 \times 3} \\
{\left[\boldsymbol{f}_{k} \otimes\right]} & 0_{3 \times 3}
\end{array}\right] .
$$

From (2), the first-order partial derivative of $\boldsymbol{\varepsilon}_{f k}\left(\boldsymbol{\varepsilon}_{o j}, \boldsymbol{\beta}_{f k}\right)$ with respect to one of the parameters is given by

$$
\left.\frac{\partial \varepsilon_{f k}\left(\varepsilon_{o j}, \boldsymbol{\beta}_{f k}\right)}{\partial x_{o j}}\right|_{0}=\left.{ }^{b f k} B_{o j} \frac{\partial \varepsilon_{o j}}{\partial x_{o j}}\right|_{0}-\left.{ }^{b f k} B_{L f k} D_{L f k} \frac{\partial \boldsymbol{\beta}_{f k}}{\partial x_{o j}}\right|_{0},
$$

where, 


$$
\begin{gathered}
D_{L f k}:=\left[\begin{array}{lll}
C_{f k} & \boldsymbol{u}_{r z} & -{ }^{L f k} B_{L o j k} C_{o j k}
\end{array}\right]=\left[\begin{array}{ccc}
M_{f k} & 0_{2 \times 1} & -{ }^{L f k} \tilde{R}_{L o j k} M_{o j k} \\
0_{1 \times 2} & 0 & 0_{1 \times 2} \\
\Omega K_{f k} M_{f k} & 0_{2 \times 1} & -{ }^{L f k} \tilde{R}_{L o j k} \Omega K_{o j k} M_{o j k} \\
T_{f k} M_{f k} & 1 & T_{o j k} M_{o j k}
\end{array}\right] \in \mathbb{R}^{6 \times 5}, \\
{ }^{L f k} R_{L o j k}=I_{34}{ }^{L f k} A_{L o j k}(0) I_{34}^{T}=\left[\begin{array}{cc}
{ }^{L f k} \tilde{R}_{L o j k} & 0_{2 \times 1} \\
0_{1 \times 2} & -1
\end{array}\right],{ }^{L f k} \tilde{R}_{L o j k}:=\left[\begin{array}{cc}
\cos \phi_{f k 0} & \sin \phi_{f k 0} \\
\sin \phi_{f k 0} & -\cos \phi_{f k 0}
\end{array}\right]={ }^{L f k} \tilde{R}_{L o j k}^{-1} .
\end{gathered}
$$

The matrices $C_{f k}$ and $C_{o j k}$ are obtained from the contact surface geometry. Note that all the elements of the third row of $D_{L f k}$ are zero. Finally, we have

$$
\left.\frac{\partial \varepsilon_{f k}\left(\varepsilon_{o j}, \boldsymbol{\beta}_{f k}\right)}{\partial \varepsilon_{o j}^{T}}\right|_{0}={ }^{b f k} B_{o j},\left.\frac{\partial \varepsilon_{f k}\left(\varepsilon_{o j}, \boldsymbol{\beta}_{f k}\right)}{\partial \boldsymbol{\beta}_{f k}^{T}}\right|_{0}=-{ }^{b f k} B_{L f k} D_{L f k}
$$

From (5), the first-order partial derivative of $U_{j k}\left(\boldsymbol{\varepsilon}_{o j}, \boldsymbol{\beta}_{f k}\right)$ is obtained by

$$
\left[\begin{array}{c}
U_{j k, \varepsilon} \\
U_{j k, \beta}
\end{array}\right]=\left[\begin{array}{l}
\left.\frac{\partial \boldsymbol{x}_{f k}^{T}\left(\boldsymbol{\varepsilon}_{o j}, \boldsymbol{\beta}_{f k}\right)}{\partial \boldsymbol{\varepsilon}_{o j}}\right|_{0} \\
\left.\frac{\partial \boldsymbol{x}_{f k}^{T}\left(\boldsymbol{\varepsilon}_{o j}, \boldsymbol{\beta}_{f k}\right)}{\partial \boldsymbol{\beta}_{f k}}\right|_{0}
\end{array}\right] \boldsymbol{f}_{k}=\left\{\boldsymbol{f}_{k}^{T} I_{3 x}\left[{ }^{b f k} B_{o j} \quad-{ }^{b f k} B_{L f k} D_{L f k}\right]\right\}^{T} .
$$

The second-order partial derivative of $U_{j k}\left(\boldsymbol{\varepsilon}_{o j}, \boldsymbol{\beta}_{f k}\right)$ is given by

$$
\begin{gathered}
{\left[\begin{array}{ll}
U_{j k, \varepsilon \varepsilon} & U_{j k, \beta \varepsilon} \\
U_{j k, \varepsilon \beta} & U_{j k, \beta \beta}
\end{array}\right]=\left[\begin{array}{l}
\left.\frac{\partial \boldsymbol{x}_{f k}^{T}\left(\boldsymbol{\varepsilon}_{o j}, \boldsymbol{\beta}_{f k}\right)}{\partial \boldsymbol{\varepsilon}_{o j}}\right|_{0} \\
\left.\frac{\partial \boldsymbol{x}_{f k}^{T}\left(\boldsymbol{\varepsilon}_{o j}, \boldsymbol{\beta}_{f k}\right)}{\partial \boldsymbol{\beta}_{f k}}\right|_{0}
\end{array} S_{k}\left[\begin{array}{l}
\left.\frac{\partial \boldsymbol{x}_{f k}^{T}\left(\boldsymbol{\varepsilon}_{o j}, \boldsymbol{\beta}_{f k}\right)}{\partial \boldsymbol{\varepsilon}_{o j}}\right|_{0} \\
\left.\frac{\partial \boldsymbol{x}_{f k}^{T}\left(\boldsymbol{\varepsilon}_{o j}, \boldsymbol{\beta}_{f k}\right)}{\partial \boldsymbol{\beta}_{f k}}\right|_{0}
\end{array}\right]^{T}+\left[\begin{array}{cc}
\left.\frac{\partial^{2}\left[\boldsymbol{f}_{k}^{T} \boldsymbol{x}_{f k}\left(\boldsymbol{\varepsilon}_{o j}, \boldsymbol{\beta}_{f k}\right)\right]}{\partial \boldsymbol{\varepsilon}_{o j} \partial \boldsymbol{\varepsilon}_{o j}^{T}}\right|_{0} & \left.\frac{\partial^{2}\left[\boldsymbol{f}_{k}^{T} \boldsymbol{x}_{f k}\left(\boldsymbol{\varepsilon}_{o j}, \boldsymbol{\beta}_{f k}\right)\right]}{\partial \boldsymbol{\varepsilon}_{o j} \partial \boldsymbol{\beta}_{f k}^{T}}\right|_{0} \\
\left.\frac{\partial^{2}\left[\boldsymbol{f}_{k}^{T} \boldsymbol{x}_{f k}\left(\boldsymbol{\varepsilon}_{o j}, \boldsymbol{\beta}_{f k}\right)\right]}{\partial \boldsymbol{\beta}_{f k} \partial \boldsymbol{\varepsilon}_{o j}^{T}}\right|_{0} & \frac{\partial^{2}\left[\boldsymbol{f}_{k}^{T} \boldsymbol{x}_{f k}\left(\boldsymbol{\varepsilon}_{o j}, \boldsymbol{\beta}_{f k}\right)\right]}{\left.\partial \boldsymbol{\beta}_{f k} \partial \boldsymbol{\beta}_{f k}^{T}\right|_{0}}
\end{array}\right]\right.} \\
=\left[\begin{array}{lll}
{ }^{L f k} B_{o j} & \left.-D_{L f k}\right]^{T}{ }^{L f k} S_{k}\left[{ }^{L f k} B_{o j}\right. & -D_{L f k}
\end{array}\right]+\left[\begin{array}{ccc}
0_{3 \times 3} & 0_{3 \times 5} \\
0_{3 \times 3} & -I_{3 \xi}{ }^{L f k} B_{o j}^{T}{ }^{L f k} F_{k}{ }^{L f k} B_{o j} I_{3 \xi}^{T} & I_{3 \xi}^{L f k} B_{o j}^{T}{ }^{L f k} F_{k} D_{L f k} \\
0_{5 \times 3} & s y m . & \left.\frac{\partial^{2}\left[\boldsymbol{f}_{k}^{T} \boldsymbol{x}_{f k}\left(\boldsymbol{\varepsilon}_{o j}, \boldsymbol{\beta}_{f k}\right)\right]}{\partial \boldsymbol{\beta}_{f k} \partial \boldsymbol{\beta}_{f k}^{T}}\right|_{0}
\end{array}\right],
\end{gathered}
$$

where

$$
\begin{aligned}
& \left.\frac{\partial^{2}\left[\boldsymbol{f}_{k}^{T} \boldsymbol{x}_{f k}\left(\boldsymbol{\varepsilon}_{o j}, \boldsymbol{\beta}_{f k}\right)\right]}{\partial \boldsymbol{\beta}_{f k} \partial \boldsymbol{\beta}_{f k}^{T}}\right|_{0}=\left[\begin{array}{ccc}
-\left[C_{f k}^{T}{ }^{L f k} F_{k} C_{f k}\right]^{T} & \text { sym. } & \text { sym. } \\
-\boldsymbol{u}_{r z}^{T}{ }^{L f k} F_{k} C_{f k} & -\boldsymbol{u}_{r z}^{T}{ }^{L f k} F_{k} \boldsymbol{u}_{r z} & \text { sym. } \\
\left.{ }^{L f k} B_{L o j k} C_{o j k}\right]^{T L f k} F_{k} D_{L f k} &
\end{array}\right] \in \mathbb{R}^{5 \times 5}, \\
& { }^{L f k} S_{k}:=\left[I_{3 x}{ }^{b f k} B_{L f k}\right]{ }^{T} S_{k}\left[I_{3 x}{ }^{b f k} B_{L f k}\right],{ }^{L f k} F_{k}:={ }^{b f k} B_{L f k}^{T} F_{k}{ }^{b f k} B_{L f k} .
\end{aligned}
$$

\section{Appendix D: Partial Derivatives of (7) and (8)}

The first-order partial derivative of (7) is given by

$$
I_{3 \xi}\left[{ }^{b f k} B_{o j}-\left.{ }^{b f k} B_{L f k} D_{L f k} \frac{\partial \boldsymbol{\beta}_{f k}^{r c}}{\partial \varepsilon_{o j}^{T}}\right|_{0}\right]=0_{3 \times 6}
$$

Using $I_{3 \xi}{ }^{a} B_{b}={ }^{a} R_{b} I_{3 \xi}$, we have

$$
I_{3 \xi} D_{L f k} \boldsymbol{\beta}_{f k, \varepsilon}^{r c}={ }^{L f k} R_{o j} I_{3 \xi} .
$$

From (7), we have the following second-order partial derivative:

$$
\begin{gathered}
\left.\boldsymbol{u}_{p}^{T} I_{3 \xi} D_{L f k} \frac{\partial^{2} \boldsymbol{\beta}_{f k}^{r c}}{\partial y_{o j} \partial x_{o j}}\right|_{0}=\boldsymbol{u}_{p}^{T}\left[\left(\left.{ }^{L f k} R_{o j} I_{3 \xi} \frac{\partial \boldsymbol{\varepsilon}_{o j}}{\partial y_{o j}}\right|_{0}\right) \otimes\left(\left.{ }^{L f k} R_{o j} I_{3 \xi} \frac{\partial \boldsymbol{\varepsilon}_{o j}}{\partial x_{o j}}\right|_{0}\right)\right]- \\
\boldsymbol{u}_{p}^{T}\left[\left(\left.I_{3 \xi} C_{f k} \frac{\partial \boldsymbol{\theta}_{f k}}{\partial y_{o j}}\right|_{0}+\left.\boldsymbol{u}_{3} \frac{\partial \phi_{f k}}{\partial y_{o j}}\right|_{0}\right) \otimes\left(\left.{ }^{L f k} R_{o j} I_{3 \xi} \frac{\partial \varepsilon_{o j}}{\partial x_{o j}}\right|_{0}\right)\right]+\boldsymbol{u}_{p}^{T}\left[\left(\left.\boldsymbol{u}_{3} \frac{\partial \phi_{f k}}{\partial y_{o j}}\right|_{0}\right) \otimes\left(\left.I_{3 \xi} C_{f k} \frac{\partial \boldsymbol{\theta}_{f k}}{\partial x_{o j}}\right|_{0}\right)\right],
\end{gathered}
$$

where, $p=1,2,3$. For all the object pose parameters, we have 


$$
\left.\frac{\partial^{2}\left[\boldsymbol{u}_{p}^{T} I_{3 \xi} D_{L f k} \boldsymbol{\beta}_{f k}^{r c}\right]}{\partial \boldsymbol{\varepsilon}_{o j} \partial \boldsymbol{\varepsilon}_{o j}^{T}}\right|_{0}=-{ }^{L f k} B_{o j}^{T}\left\{I_{3 \xi}^{T}\left[\boldsymbol{u}_{p} \otimes\right] I_{3 \xi}\right\}^{L f k} B_{o j}+
$$

$\left\{\left[\begin{array}{lll}C_{f k} & \boldsymbol{u}_{r z} & 0_{6 \times 2}\end{array}\right] \boldsymbol{\beta}_{f k, \varepsilon}\right\}^{T}\left\{I_{3 \xi}^{T}\left[\boldsymbol{u}_{p} \otimes\right] I_{3 \xi}\right\}^{L f k} B_{o j}-\left\{\left[\begin{array}{lll}0_{6 \times 2} & \boldsymbol{u}_{r z} & 0_{6 \times 2}\end{array}\right] \boldsymbol{\beta}_{f k, \varepsilon}\right\}^{T}\left\{I_{3 \xi}^{T}\left[\boldsymbol{u}_{p} \otimes\right] I_{3 \xi}\right\}\left[C_{f k} \quad 0_{6 \times 1} \quad 0_{6 \times 2}\right] \boldsymbol{\beta}_{f k, \varepsilon}$

For infinitesimal displacements, (8) is satisfied with respect to all the object pose parameters. Hence, we have

$$
I_{24}\left[\left.{ }^{L f k} A_{L o j k}\left(\boldsymbol{\beta}_{f k}\right) \frac{\partial^{L f k} A_{L o j k}\left(\boldsymbol{\beta}_{f k}\right)}{\partial x_{o j}}\right|_{0}\right] \boldsymbol{v}_{p}=\left.I_{26} D_{L f k} \frac{\partial \boldsymbol{\beta}_{f k}^{f r}}{\partial x_{o j}}\right|_{0}=0_{2 \times 1} .
$$

Finally, we have

$$
\left.I_{26} D_{L f k} \frac{\partial \boldsymbol{\beta}_{f k}^{f r}}{\partial \varepsilon_{o j}^{T}}\right|_{0}=0_{2 \times 6} .
$$

From (8), we have the following second-order partial derivative:

$$
\left.\frac{\partial^{2}\left[\boldsymbol{u}_{p}^{T} I_{3 x} D_{L f k} \boldsymbol{\beta}_{f k}^{f r}\right]}{\partial y_{o j} \partial x_{o j}}\right|_{0}=\boldsymbol{u}_{p}^{T}\left[\left(I_{3 x} C_{f k} \frac{\partial \boldsymbol{\theta}_{f k}}{\partial y_{o j}} I_{0}\right) \otimes\left(\left.{ }^{L f k} R_{o j} I_{3 \xi} \frac{\partial \boldsymbol{\varepsilon}_{o j}}{\partial x_{o j}}\right|_{0}\right)\right]-\boldsymbol{u}_{p}^{T}\left[\left(\boldsymbol{u}_{3} \frac{\partial \phi_{f k}}{\partial y_{o j}} I_{0}\right) \otimes\left(I_{3 x} C_{f k} \frac{\partial \boldsymbol{\theta}_{f k}}{\partial x_{o j}} I_{0}\right)\right],
$$

where $p=1,2$. Finally, we have

$$
\begin{aligned}
& \left.\frac{\partial^{2}\left[\boldsymbol{u}_{p}^{T} I_{3 x} D_{L f k} \boldsymbol{\beta}_{f k}^{f r}\right.}{\partial \boldsymbol{\varepsilon}_{o j} \partial \boldsymbol{\varepsilon}_{o j}^{T}}\right|_{0}=\left\{\left[\begin{array}{lll}
C_{f k} & 0_{6 \times 1} & 0_{6 \times 2}
\end{array}\right] \boldsymbol{\beta}_{f k, \varepsilon}\right\}^{T}\left\{I_{3 x}^{T}\left[\boldsymbol{u}_{p} \otimes\right] I_{3 \xi}\right\}^{L f k} B_{o j}- \\
& \left\{\left[\begin{array}{lll}
0_{6 \times 2} & \boldsymbol{u}_{r z} & 0_{6 \times 2}
\end{array}\right] \boldsymbol{\beta}_{f k, \varepsilon}\right\}^{T}\left\{I_{3 \xi}^{T}\left[\boldsymbol{u}_{p} \otimes\right] I_{3 x}\right\}\left[\begin{array}{lll}
C_{f k} & 0_{6 \times 1} & 0_{6 \times 2}
\end{array}\right] \boldsymbol{\beta}_{f k, \varepsilon} .
\end{aligned}
$$

\section{Appendix E: Derivations of (11)}

The inverse matrix included in (9) is expanded as

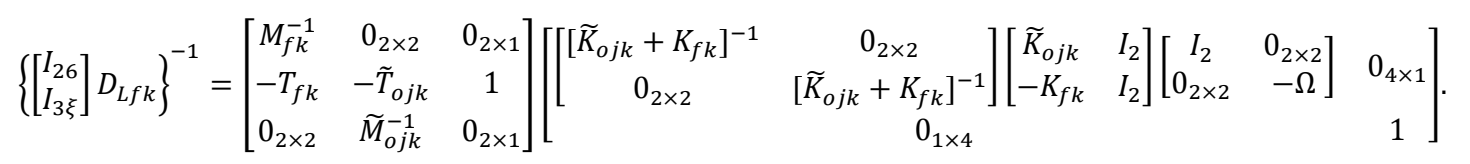

Because the matrix $\widetilde{K}_{o j k}+K_{f k}$ is positive definite from (A2), $\widetilde{K}_{o j k}+K_{f k}$ is invertible and then the inverse matrix (90) exists. The first-order partial derivative of $U_{j k}^{f r}\left(\boldsymbol{\varepsilon}_{o j}\right)$ is obtained by

$$
\begin{aligned}
& {\left[G_{j k}^{f r}\right]^{T}=U_{j k, \varepsilon}^{T}+U_{j k, \beta}^{T} \boldsymbol{\beta}_{f k, \varepsilon}^{f r}=\boldsymbol{f}_{k}^{T} I_{3 x}{ }^{b f k} B_{o j}-\boldsymbol{f}_{k}^{T} I_{3 x}{ }^{b f k} B_{L f k} D_{L f k}\left\{\left[\begin{array}{c}
I_{26} \\
I_{3 \xi}
\end{array}\right] D_{L f k}\right\}^{-1}\left[\begin{array}{c}
0_{2 \times 3} \\
I_{3}
\end{array}\right]^{L f k} R_{o j} I_{3 \xi}} \\
& =\boldsymbol{f}_{k}^{T} I_{3 x}{ }^{b f k} B_{L f k}\left[{ }^{L f k} B_{o j}-\left\{\left[\begin{array}{l}
I_{26} \\
I_{3 \xi}
\end{array}\right]^{T}\left[\begin{array}{c}
I_{26} \\
I_{3 \xi}
\end{array}\right]+\left[\begin{array}{c}
\boldsymbol{u}_{3} \\
0_{3 \times 1}
\end{array}\right]\left[\begin{array}{c}
\boldsymbol{u}_{3} \\
0_{3 \times 1}
\end{array}\right]^{T}\right\} D_{L f k}\left\{\left[\begin{array}{l}
I_{26} \\
I_{3 \xi}
\end{array} D_{L f k}\right\}^{-1}\left[\begin{array}{c}
0_{2 \times 3} \\
I_{3}
\end{array}\right]_{3 \xi}{ }^{L f k} B_{o j}\right]\right. \\
& =\boldsymbol{f}_{k}^{T} I_{3 x}{ }^{b f k} B_{L f k}\left[I_{6}-I_{3 \xi}^{T} I_{3 \xi}\right]^{L f k} B_{o j}=\boldsymbol{f}_{k}^{T}\left[I_{3 x}{ }^{b f k} B_{L f k} I_{3 x}^{T}\right]\left[I_{3 x}{ }^{L f k} B_{o j}\right]=\left[{ }^{o j} W_{L f k}{ }^{L f k} \boldsymbol{f}_{k}\right]^{T},
\end{aligned}
$$

where note that $\left[\begin{array}{ll}\boldsymbol{u}_{3}^{T} & 0_{1 \times 3}\end{array}\right] D_{L f k}=0_{1 \times 5}$. The last term of $H_{j k}^{f r}$ is given by

$$
\begin{gathered}
\left.\frac{\partial^{2}\left[U_{j k, \beta}^{T} \boldsymbol{\beta}_{f k}^{f r}\left(\boldsymbol{\varepsilon}_{o j}\right)\right]}{\partial y_{o j} \partial x_{o j}}\right|_{0}=-\left.\boldsymbol{f}_{k}^{T} I_{3 x}{ }^{b f k} B_{L f k} D_{L f k} \frac{\partial^{2} \boldsymbol{\beta}_{f k}^{f r}\left(\boldsymbol{\varepsilon}_{o j}\right)}{\partial y_{o j} \partial x_{o j}}\right|_{0} \\
=-\boldsymbol{f}_{k}^{T} I_{3 x}{ }^{b f k} B_{L f k}\left\{\left[\begin{array}{l}
I_{26} \\
I_{3 \xi}
\end{array}\right]^{T}\left[\begin{array}{c}
I_{26} \\
I_{3 \xi}
\end{array}\right]+\left[\begin{array}{c}
\boldsymbol{u}_{3} \\
0_{3 \times 1}
\end{array}\right]\left[\begin{array}{c}
\boldsymbol{u}_{3} \\
0_{3 \times 1}
\end{array}\right]^{T}\right\} D_{L f k}\left\{\left[\begin{array}{c}
I_{26} \\
I_{3 \xi}
\end{array}\right] D_{L f k}\right\}^{-1} \times \\
I_{23}\left[\left(I_{3 x} C_{f k} \frac{\partial \boldsymbol{\theta}_{f k}}{\partial y_{o j}} l_{0}\right) \otimes\left({ }^{L f k} R_{o j} I_{3 \xi} \frac{\partial \varepsilon_{o j}}{\partial x_{o j}} l_{0}\right)-\left(\boldsymbol{u}_{3} \frac{\partial \phi_{f k}}{\partial y_{o j}} l_{0}\right) \otimes\left(I_{3 x} C_{f k} \frac{\partial \boldsymbol{\theta}_{f k}}{\partial x_{o j}} l_{0}\right)\right] \\
\left.\left(I_{3 \xi} C_{f k} \frac{\partial \boldsymbol{\theta}_{f k}}{\partial y_{o j}} l_{0}+\boldsymbol{u}_{3} \frac{\partial \phi_{f k}}{\partial y_{o j}} l_{0}-{ }^{L f k} R_{o j} I_{3 \xi} \frac{\partial \varepsilon_{o j}}{\partial y_{o j}} l_{0}\right) \otimes\left({ }^{L f k} R_{o j} I_{3 \xi} \frac{\partial \boldsymbol{\varepsilon}_{o j}}{\partial x_{o j}} l_{0}\right)-\left(\boldsymbol{u}_{3} \frac{\partial \phi_{f k}}{\partial y_{o j}} l_{0}\right) \otimes\left(I_{3 \xi} C_{f k} \frac{\partial \boldsymbol{\theta}_{f k}}{\partial x_{o j}} l_{0}\right)\right] .
\end{gathered}
$$

Finally, we have

$$
\left.\frac{\partial^{2}\left[U_{j k, \beta}^{T} \boldsymbol{\beta}_{f k}^{f r}\left(\boldsymbol{\varepsilon}_{o j}\right)\right]}{\partial \boldsymbol{\varepsilon}_{o j} \partial \boldsymbol{\varepsilon}_{o j}^{T}}\right|_{0}={ }^{L f k} B_{o j}^{T}\left[\begin{array}{cc}
0_{3 \times 3} & 0_{3 \times 3} \\
0_{3 \times 3} & {\left[\left({ }^{L f k} \boldsymbol{p}_{b f k} \otimes{ }^{L f k} \boldsymbol{f}_{k}\right) \otimes\right]}
\end{array}{ }^{L f k} B_{o j}-\right.
$$




$$
\begin{aligned}
& \left\{\left[\begin{array}{lll}
C_{f k} & \boldsymbol{u}_{r z} & 0_{6 \times 2}
\end{array}\right] \boldsymbol{\beta}_{f k, \varepsilon}^{f r}\right\}^{T}\left[\begin{array}{cc}
0_{3 \times 3} & {\left[\left(I_{23}^{T} I_{23}{ }^{L f k} \boldsymbol{f}_{k}\right) \otimes\right]} \\
0_{3 \times 3} & {\left[\left({ }^{L f k} \boldsymbol{p}_{b f k} \otimes{ }^{L f k} \boldsymbol{f}_{k}\right) \otimes\right]}
\end{array}\right]{ }^{L f k} B_{o j}+
\end{aligned}
$$

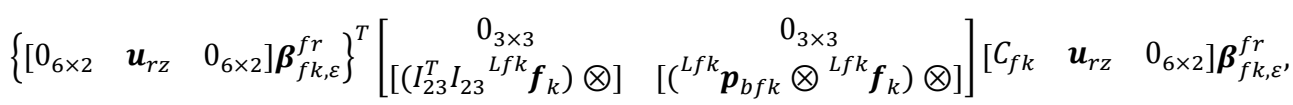

where

$$
\begin{gathered}
\boldsymbol{f}_{k}^{T} I_{3 x}{ }^{b f k} B_{L f k} I_{3 x}^{T} \boldsymbol{u}_{p}=\boldsymbol{f}_{k}^{T b f k} R_{L f k} \boldsymbol{u}_{p}=\boldsymbol{u}_{p}^{T L f k} \boldsymbol{f}_{k}, \\
\boldsymbol{f}_{k}^{T} I_{3 x}{ }^{b f k} B_{L f k} I_{3 \xi}^{T} \boldsymbol{u}_{p}=\boldsymbol{f}_{k}^{T}\left[{ }^{b f k} \boldsymbol{p}_{L f k} \otimes{ }^{b f k} R_{L f k}\right] \boldsymbol{u}_{p}=-\boldsymbol{f}_{k}^{T b f k} R_{L f k}\left[{ }^{L f k} \boldsymbol{p}_{b f k} \otimes\right] \boldsymbol{u}_{p}=\boldsymbol{u}_{p}^{T}\left[{ }^{L f k} \boldsymbol{p}_{b f k} \otimes\right]^{L f k} \boldsymbol{f}_{k}
\end{gathered}
$$

\section{Appendix F: Derivations of (15)}

From the partial derivative of $\boldsymbol{\gamma}_{k}$, we have the following conditions:

$$
\begin{gathered}
\left.\frac{\partial \boldsymbol{\gamma}_{k}}{\partial \boldsymbol{\varepsilon}_{o j}^{T}}\right|_{0}=\left.\frac{\partial\left[I_{\gamma k} \boldsymbol{\beta}_{f k}^{r c}\left(\boldsymbol{\varepsilon}_{o j}, \boldsymbol{\gamma}_{k}\right)\right]}{\partial \boldsymbol{\varepsilon}_{o j}^{T}}\right|_{0}=I_{\gamma k} \boldsymbol{\beta}_{f k, \varepsilon}^{r c}=0_{2 \times 6}, \\
\left.\frac{\partial \boldsymbol{\gamma}_{k}}{\partial \boldsymbol{\gamma}_{k}^{T}}\right|_{0}=\left.\frac{\partial\left[I_{\gamma k} \boldsymbol{\beta}_{f k}^{r c}\left(\boldsymbol{\varepsilon}_{o}, \boldsymbol{\gamma}_{k}\right)\right]}{\partial \boldsymbol{\gamma}_{k}^{T}}\right|_{0}=I_{\gamma k} \boldsymbol{\beta}_{f k, \gamma}^{r c}=I_{2} .
\end{gathered}
$$

The left-hand side of (83) is rewritten as

$$
I_{3 \xi} D_{L f k} \boldsymbol{\beta}_{f k, \varepsilon}^{r c}=I_{3 \xi} D_{L f k}\left[I_{\gamma k}^{T} I_{\gamma k}+I_{r c k}^{T} I_{r c k}\right] \boldsymbol{\beta}_{f k, \varepsilon}^{r c}=P_{d k} I_{r c k} \boldsymbol{\beta}_{f k, \varepsilon}^{r c} .
$$

If $P_{d k}$ is invertible, we have

$$
I_{r c k} \boldsymbol{\beta}_{f k, \varepsilon}^{r c}=P_{d k}^{-1 L f k} R_{o j} I_{3 \xi}
$$

Finally, we have

$$
\boldsymbol{\beta}_{f k, \varepsilon}^{r c}=\left[I_{\gamma k}^{T} I_{\gamma k}+I_{r c k}^{T} I_{r c k}\right] \boldsymbol{\beta}_{f k, \varepsilon}^{r c}=I_{r c k}^{T} P_{d k}^{-1}{ }^{L f k} R_{o j} I_{3 \xi}
$$

From (14), we have

$$
I_{3 \xi}^{b f k} B_{L f k} D_{L f k} \boldsymbol{\beta}_{f k, \gamma}^{r c}=0_{3 \times 2}
$$

This means

$$
I_{3 \xi} D_{L f k}\left[I_{\gamma k}^{T} I_{\gamma k}+I_{r c k}^{T} I_{r c k}\right] \boldsymbol{\beta}_{f k, \gamma}^{r c}=0_{3 \times 2}
$$

and then

Finally, we have

$$
I_{r c k} \boldsymbol{\beta}_{f k, \gamma}^{r c}=-P_{d k}^{-1} I_{3 \xi} D_{L f k} I_{\gamma k}^{T}
$$

$$
\boldsymbol{\beta}_{f k, \gamma}^{r c}=\left[I_{\gamma k}^{T} I_{\gamma k}+I_{r c k}^{T} I_{r c k}\right] \boldsymbol{\beta}_{f k, \gamma}^{r c}=I_{\gamma k}^{T}-I_{r c k}^{T} P_{d k}^{-1} I_{3 \xi} D_{L f k} I_{\gamma k}^{T} .
$$

In the case described in Section 3.5.1.3, we derive the matrices $P_{\gamma k}$ and $P_{d k}$. The matrices $I_{\gamma k}$ and $I_{r c k}$ provided in (21) satisfy $I_{\gamma k} I_{r c k}^{T}=0_{2 \times 3}$. The square matrix $P_{\gamma k} \in \mathbb{R}^{2 \times 2}$ has to satisfy the following condition:

$$
I_{2}=I_{\gamma k} I_{\gamma k}^{T}=P_{\gamma k}\left[M_{f k} M_{f k}^{T}+\widetilde{M}_{o j k} \widetilde{M}_{o j k}^{T}\right] P_{\gamma k}^{T}
$$

Finally, we have (22). In this case,

$$
\begin{aligned}
& I_{3 x} D_{L f k} I_{r c k}^{T}=0_{3 \times 3}, \\
& P_{d k}=\left[\begin{array}{ccc}
\Omega K_{f k} M_{f k} & 0_{2 \times 1} & -{ }^{L f k} \tilde{R}_{L o j k} \Omega K_{o j k} M_{o j k} \\
T_{f k} M_{f k} & 1 & T_{o j k} M_{o j k}
\end{array}\right]\left[\begin{array}{cc}
M_{f k}^{-1} & 0_{2 \times 1} \\
0_{1 \times 2} & 1 \\
{\left[{ }^{L f k} \tilde{R}_{L o j k} M_{o j k}\right]^{-1}} & 0_{2 \times 1}
\end{array}\right]\left[\begin{array}{cc}
P_{\gamma k}^{-T} & 0_{2 \times 1} \\
0_{1 \times 2} & 1
\end{array}\right] \\
& =\left[\begin{array}{cc}
{\left[\Omega K_{f k}-{ }^{L f k} \tilde{R}_{L o j k} \Omega K_{o j k}{ }^{L f k} \tilde{R}_{L o j k}^{-1}\right] P_{\gamma k}^{-T}} & 0_{2 \times 1} \\
{\left[T_{f k}+T_{o j k}{ }^{L f k} \tilde{R}_{L o j k}^{-1}\right] P_{\gamma k}^{-T}} & 1
\end{array}\right]=\left[\begin{array}{cc}
\Omega\left[K_{f k}+\widetilde{K}_{o j k}\right] P_{\gamma k}^{-T} & 0_{2 \times 1} \\
{\left[T_{f k}+\tilde{T}_{o j k}\right] P_{\gamma k}^{-T}} & 1
\end{array}\right],
\end{aligned}
$$

where

$$
{ }^{L f k} \tilde{R}_{L o j k} \Omega K_{o j k}{ }^{L f k} \tilde{R}_{L o j k}^{-1}=\left[{ }^{L f k} \tilde{R}_{L o j k} \Omega^{L f k} \tilde{R}_{L o j k}^{-1}\right]\left[{ }^{L f k} \tilde{R}_{L o j k} K_{o j k}{ }^{L f k} \tilde{R}_{L o j k}^{-1}\right]=-\Omega \widetilde{K}_{o j k}
$$




\section{Appendix G: Derivations of (28)}

From (27), we have

$$
\begin{aligned}
& {\left[\begin{array}{c}
U_{j k, \varepsilon}^{r c} \\
U_{j k, \gamma}^{r c}
\end{array}\right]=\left[\begin{array}{cc}
I_{6} & 0_{6 \times 2} \\
\boldsymbol{\beta}_{f k, \varepsilon}^{r c} & \boldsymbol{\beta}_{f k, \gamma}^{r c}
\end{array}\right]^{T}\left[\begin{array}{c}
U_{j k, \varepsilon} \\
U_{j k, \beta}
\end{array}\right]} \\
& {\left[\begin{array}{cc}
U_{j k, \varepsilon \varepsilon}^{r c} & U_{j k, \gamma \varepsilon}^{r c} \\
U_{j k, \varepsilon \gamma}^{r c} & U_{j k, \gamma \gamma}^{r c}
\end{array}\right]=\left[\begin{array}{cc}
I_{6} & 0_{6 \times 2} \\
\boldsymbol{\beta}_{f k, \varepsilon}^{r c} & \boldsymbol{\beta}_{f k, \gamma}^{r c}
\end{array}\right]^{T}\left[\begin{array}{cc}
U_{j k, \varepsilon \varepsilon} & U_{j k, \beta \varepsilon} \\
U_{j k, \varepsilon \beta} & U_{j k, \beta \beta}
\end{array}\right]\left[\begin{array}{cc}
I_{6} & 0_{6 \times 2} \\
\boldsymbol{\beta}_{f k, \varepsilon}^{r c} & \boldsymbol{\beta}_{f k, \gamma}^{r c}
\end{array}\right]+} \\
& {\left[\begin{array}{ll}
\left.\frac{\partial^{2}\left[U_{j k, \beta}^{T} \boldsymbol{\beta}_{f k}^{r c}\left(\boldsymbol{\varepsilon}_{o j}, \boldsymbol{\gamma}_{k}\right)\right]}{\partial \boldsymbol{\varepsilon}_{o j} \partial \boldsymbol{\varepsilon}_{o j}^{T}}\right|_{0} & \left.\frac{\partial^{2}\left[U_{j k, \beta}^{T} \boldsymbol{\beta}_{f k}^{r c}\left(\boldsymbol{\varepsilon}_{o j}, \boldsymbol{\gamma}_{k}\right)\right]}{\partial \boldsymbol{\varepsilon}_{o j} \partial \boldsymbol{\gamma}_{k}^{T}}\right|_{0} \\
\left.\frac{\partial^{2}\left[U_{j k, \beta}^{T} \boldsymbol{\beta}_{f k}^{r c}\left(\boldsymbol{\varepsilon}_{o j}, \boldsymbol{\gamma}_{k}\right)\right]}{\partial \boldsymbol{\gamma}_{k} \partial \boldsymbol{\varepsilon}_{o j}^{T}}\right|_{0} & \left.\frac{\partial^{2}\left[U_{j k, \beta}^{T} \boldsymbol{\beta}_{f k}^{r c}\left(\boldsymbol{\varepsilon}_{o j}, \boldsymbol{\gamma}_{k}\right)\right]}{\partial \boldsymbol{\gamma}_{k} \partial \boldsymbol{\gamma}_{k}^{T}}\right|_{0}
\end{array}\right]} \\
& =\left[\begin{array}{cc}
I_{6} & 0_{6 \times 2} \\
\boldsymbol{\beta}_{f k, \varepsilon}^{r c} & \boldsymbol{\beta}_{f k, \gamma}^{r c}
\end{array}\right]^{T}\left[\begin{array}{cc}
U_{j k, \varepsilon \varepsilon} & U_{j k, \beta \varepsilon} \\
U_{j k, \varepsilon \beta} & U_{j k, \beta \beta}
\end{array}\right]\left[\begin{array}{cc}
I_{6} & 0_{6 \times 2} \\
\boldsymbol{\beta}_{f k, \varepsilon}^{r c} & \boldsymbol{\beta}_{f k, \gamma}^{r c}
\end{array}\right]+
\end{aligned}
$$

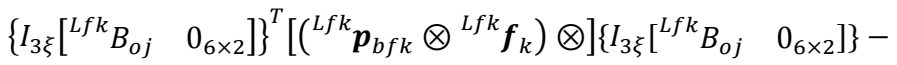

$$
\begin{aligned}
& \left.\left\{\begin{array}{lll}
\left.I_{3 \xi}\left[\begin{array}{lll}
C_{f k} & \boldsymbol{u}_{r z} & 0_{6 \times 2}
\end{array}\right]\left[\begin{array}{ll}
\boldsymbol{\beta}_{f k, \varepsilon}^{r c} & \boldsymbol{\beta}_{f k, \gamma}^{r c}
\end{array}\right]\right\}^{T}\left[\left({ }^{L f k} \boldsymbol{p}_{b f k} \otimes{ }^{L f k} \boldsymbol{f}_{k}\right) \otimes\right]\left\{I _ { 3 \xi } \left[{ }^{L f k} B_{o j}\right.\right. & 0_{6 \times 2}
\end{array}\right]\right\}+
\end{aligned}
$$

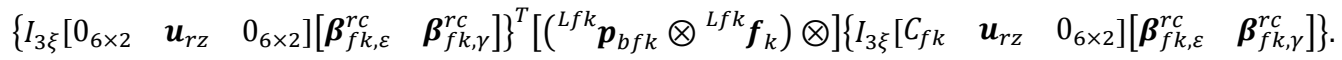

\section{Appendix H: Derivations of (36)}

From (22), (79), (102), and (106), we have

$$
\begin{gathered}
{\left[U_{j k, \gamma}^{r c}\right]^{T}=U_{j k, \beta}^{T} \boldsymbol{\beta}_{f k, \gamma}^{r c}=-\boldsymbol{f}_{k}^{T} I_{3 x}{ }^{b f k} B_{L f k} D_{L f k} \boldsymbol{\beta}_{f k, \gamma}^{r c}=-\boldsymbol{f}_{k}^{T} I_{3 x}{ }^{b f k} B_{L f k}\left[I_{3 x}^{T} I_{3 x}+I_{3 \xi}^{T} I_{3 \xi}\right] D_{L f k} \boldsymbol{\beta}_{f k, \gamma}^{r c}} \\
=-\boldsymbol{f}_{k}^{T}\left[I_{3 x}{ }^{b f k} B_{L f k} I_{3 x}^{T}\right] I_{3 x} D_{L f k} \boldsymbol{\beta}_{f k, \gamma}^{r c}=-{ }^{L f k} \boldsymbol{f}_{k}^{T} I_{3 x} D_{L f k}\left[I_{\gamma k}^{T}-I_{r c k}^{T} P_{d k}^{-1} I_{3 \xi} D_{L f k} I_{\gamma k}^{T}\right]=-{ }^{L f k} \boldsymbol{f}_{k}^{T} I_{3 x} D_{L f k} I_{\gamma k}^{T} \\
=-{ }^{L f k} \boldsymbol{f}_{k}^{T} I_{3 x} D_{L f k}\left[P_{\gamma k} I_{26} D_{L f k}\right]^{T}=-{ }^{L f k} \boldsymbol{f}_{k}^{T} I_{23}^{T}\left[P_{\gamma k}^{T} P_{\gamma k}\right]^{-1} P_{\gamma k}^{T}=-{ }^{L f k} \boldsymbol{f}_{k}^{T} I_{23}^{T} P_{\gamma k}^{-1} .
\end{gathered}
$$

From (29) and (107), we have $I_{23}{ }^{L f k} \boldsymbol{f}_{k}=0_{2 \times 1}$ and then (31). From (29), we have

$$
\frac{\partial}{\partial \varepsilon_{o j}}\left[\frac{\partial U_{j k}^{r c}\left(\varepsilon_{o j}, \gamma_{k}^{f S}\left(\varepsilon_{o j}\right)\right)}{\partial \gamma_{k}^{T}}\right]=\frac{\partial^{2} U_{j k}^{r c}\left(\varepsilon_{o j}, \gamma_{k}\right)}{\partial \varepsilon_{o j} \partial \gamma_{k}^{T}}+\frac{\partial\left[\gamma_{k}^{f s}\left(\varepsilon_{o j}\right)\right]^{T}}{\partial \varepsilon_{o j}}\left[\frac{\partial^{2} U_{j k}^{r c}\left(\varepsilon_{o j}, \gamma_{k}\right)}{\partial \gamma_{k} \partial \gamma_{k}^{T}}\right]=0_{2 \times 1} .
$$

At the initial condition, we have (35). From (34), we have the following partial derivative:

$$
G_{j k}^{f s}=U_{j k, \varepsilon}^{r c}+\left[\boldsymbol{\gamma}_{k, \varepsilon}^{f s}\right]^{T} U_{j k, \gamma}^{r c} .
$$

Considering (29), (79) and (98), we have

$$
\begin{gathered}
{\left[G_{j k}^{f s}\right]^{T}=\left[U_{j k, \varepsilon}^{r c}\right]^{T}=U_{j k, \varepsilon}^{T}+U_{j k, \beta}^{T} \boldsymbol{\beta}_{f k, \varepsilon}^{r c}=\boldsymbol{f}_{k}^{T} I_{3 x}{ }^{b f k} B_{o j}-\boldsymbol{f}_{k}^{T} I_{3 x}{ }^{b f k} B_{L f k} D_{L f k} I_{r c k}^{T} P_{d k}^{-1}\left[{ }^{L f k} R_{o j} I_{3 \xi}\right]} \\
=\boldsymbol{f}_{k}^{T} I_{3 x}{ }^{b f k} B_{L f k}\left[I_{3 x}^{T} I_{3 x}+I_{3 \xi}^{T} I_{3 \xi}\right]^{L f k} B_{o j}-\boldsymbol{f}_{k}^{T}\left[I_{3 x}{ }^{b f k} B_{L f k}\right]\left[I_{3 x}^{T} I_{3 x}+I_{3 \xi}^{T} I_{3 \xi}\right] D_{L f k} I_{r c k}^{T} P_{d k}^{-1}\left[{ }^{L f k} R_{o j} I_{3 \xi}\right] \\
=\boldsymbol{f}_{k}^{T}\left[I_{3 x}{ }^{b f k} B_{L f k} I_{3 x}^{T}\right]\left[I_{3 x}{ }^{L f k} B_{o j}\right]=\left[{ }^{o j} W_{L f k}{ }^{L f k} \boldsymbol{f}_{k}\right]^{T} .
\end{gathered}
$$

From (34) and (35), the second-order partial derivative is given by

$$
\begin{aligned}
H_{j k}^{f s} & :=\left.\frac{\partial^{2} U_{j k}^{f s}\left(\boldsymbol{\varepsilon}_{o j}\right)}{\partial \boldsymbol{\varepsilon}_{o j} \partial \boldsymbol{\varepsilon}_{o j}^{T}}\right|_{0}=\left[\begin{array}{c}
I_{6} \\
\boldsymbol{\gamma}_{k, \varepsilon}^{f s}
\end{array}\right]^{T}\left[\begin{array}{cc}
U_{j k, \varepsilon \varepsilon}^{r c} & U_{j k, \gamma \varepsilon}^{r c} \\
U_{j k, \varepsilon \gamma}^{r c} & U_{j k, \gamma \gamma}^{r c}
\end{array}\right]\left[\begin{array}{c}
I_{6} \\
\boldsymbol{\gamma}_{k, \varepsilon}^{f s}
\end{array}\right]+\left[\left.\frac{\partial^{2}\left\{U_{j k, \gamma}^{T} \boldsymbol{\gamma}_{k}^{f s}\left(\boldsymbol{\varepsilon}_{o j}\right)\right\}}{\partial \boldsymbol{\varepsilon}_{o j} \partial \boldsymbol{\varepsilon}_{o j}^{T}}\right|_{0}\right] \\
& =U_{j k, \varepsilon \varepsilon}^{r c}+U_{j k, \gamma \varepsilon}^{r c}\left\{-\left[U_{j k, \gamma \gamma}^{r c}\right]^{-1} U_{j k, \varepsilon \gamma}^{r c}\right\}+\left\{-\left[U_{j k, \gamma \gamma}^{r c}\right]^{-1} U_{j k, \varepsilon \gamma}^{r c}\right\}^{T} U_{j k, \varepsilon \gamma}^{r c}+ \\
& \left\{-\left[U_{j k, \gamma \gamma}^{r c}\right]^{-1} U_{j k, \varepsilon \gamma}^{r c}\right\}^{T} U_{j k, \gamma \gamma}^{r c}\left\{-\left[U_{j k, \gamma \gamma}^{r c}\right]^{-1} U_{j k, \varepsilon \gamma}^{r c}\right\}=U_{j k, \varepsilon \varepsilon}^{r c}-U_{j k, \gamma \varepsilon}^{r c}\left[U_{j k, \gamma \gamma}^{r c}\right]^{-1} U_{j k, \varepsilon \gamma}^{r c} .
\end{aligned}
$$




\section{Appendix I: External Force Effect}

When the external force is applied by the gravity of object mass, its potential energy is calculated by

$$
U_{e j}\left(\boldsymbol{\varepsilon}_{o j}\right):=-{ }^{b} \boldsymbol{p}_{g j}^{T}\left(\boldsymbol{\varepsilon}_{o j}\right)\left[m_{j}{ }^{b} \boldsymbol{g}\right],
$$

where

$$
{ }^{b} \boldsymbol{p}_{g j}\left(\boldsymbol{\varepsilon}_{o j}\right)=I_{34}{ }^{b} A_{o j}\left(\varepsilon_{o j}\right)^{o j} A_{g j} \boldsymbol{v}_{p}
$$

The frame $\Sigma_{g j}$ is the center of the object mass. The symbol $m_{j}$ is the object mass. The symbol ${ }^{b} \boldsymbol{g}=-g \boldsymbol{u}_{3}$ is the gravity vector and $g$ is the gravity acceleration. The first- and the second-order partial derivatives are obtained by the following forms:

$$
G_{e j}=-{ }^{o j} W_{g j}{ }^{g j} \boldsymbol{f}_{m g}, H_{e j}=-\left[\begin{array}{cc}
0_{3 \times 3} & 0_{3 \times 3} \\
0_{3 \times 3} & {\left[\left({ }^{o j} R_{g j}{ }^{g j} \boldsymbol{f}_{m g}\right) \otimes\right]\left[{ }^{o j} \boldsymbol{p}_{g j} \otimes\right]}
\end{array}\right],{ }^{g j} \boldsymbol{f}_{m g}:=m_{j}{ }^{g j} R_{b}{ }^{b} \boldsymbol{g} .
$$

\section{Appendix J: Partial derivatives of (41)}

The first-order partial derivative of (41) is given by the following form:

$$
\left[\begin{array}{c}
U_{i j, \varepsilon}^{o S} \\
U_{i j, \beta}^{o s}
\end{array}\right]:=\left[\begin{array}{l}
\left.\frac{\partial U_{i j}^{o S}\left(\varepsilon_{o i}, \boldsymbol{\beta}_{o i j}\right)}{\partial \varepsilon_{o i}}\right|_{0} \\
\left.\frac{\partial U_{i j}^{o S}\left(\varepsilon_{o i}, \boldsymbol{\beta}_{o i j}\right)}{\partial \boldsymbol{\beta}_{o i j}}\right|_{0}
\end{array}\right]=\left[\begin{array}{l}
\left.\frac{\partial \varepsilon_{o j}^{T}\left(\varepsilon_{o i}, \boldsymbol{\beta}_{o i j}\right)}{\partial \varepsilon_{o i}}\right|_{0} \\
\left.\frac{\partial \varepsilon_{o j}^{T}\left(\varepsilon_{o i}, \boldsymbol{\beta}_{o i j}\right)}{\partial \boldsymbol{\beta}_{o i j}}\right|_{0}
\end{array}\right] U_{j, \varepsilon}=\left[\begin{array}{ll}
{ }^{o j} B_{o i} & { }^{o j} B_{L o i j} D_{L o i j}
\end{array}\right]^{T} U_{j, \varepsilon}
$$

The second-order partial derivative is given by

$$
\begin{aligned}
& {\left[\begin{array}{ll}
U_{i j, \varepsilon \varepsilon}^{o s} & U_{i j, \beta \varepsilon}^{o s} \\
U_{i j, \varepsilon \beta}^{o s} & U_{i j, \beta \beta}^{o s}
\end{array}\right]:=\left[\begin{array}{ll}
\left.\frac{\partial^{2} U_{i j}^{o s}\left(\boldsymbol{\varepsilon}_{o i}, \boldsymbol{\beta}_{o i j}\right)}{\partial \boldsymbol{\varepsilon}_{o i} \partial \boldsymbol{\varepsilon}_{o i}^{T}}\right|_{0} & \left.\frac{\partial^{2} U_{i j}^{o s}\left(\boldsymbol{\varepsilon}_{o i}, \boldsymbol{\beta}_{o i j}\right)}{\partial \boldsymbol{\varepsilon}_{o i} \partial \boldsymbol{\beta}_{o i j}^{T}}\right|_{0} \\
\left.\frac{\partial^{2} U_{i j}^{o s}\left(\boldsymbol{\varepsilon}_{o i}, \boldsymbol{\beta}_{o i j}\right)}{\partial \boldsymbol{\beta}_{o i j} \partial \boldsymbol{\varepsilon}_{o i}^{T}}\right|_{0} & \left.\frac{\partial^{2} U_{i j}^{o s}\left(\boldsymbol{\varepsilon}_{o i}, \boldsymbol{\beta}_{o i j}\right)}{\partial \boldsymbol{\beta}_{o i j} \partial \boldsymbol{\beta}_{o i j}^{T}}\right|_{0}
\end{array}\right]} \\
& =\left[\begin{array}{l}
\left.\frac{\partial \boldsymbol{\varepsilon}_{o j}^{T}\left(\boldsymbol{\varepsilon}_{o i}, \boldsymbol{\beta}_{o i j}\right)}{\partial \boldsymbol{\varepsilon}_{o i}}\right|_{0} \\
\left.\frac{\partial \boldsymbol{\varepsilon}_{o j}^{T}\left(\boldsymbol{\varepsilon}_{o i}, \boldsymbol{\beta}_{o i j}\right)}{\partial \boldsymbol{\beta}_{o i j}}\right|_{0}
\end{array}\right]^{T} U_{j, \varepsilon \varepsilon}\left[\begin{array}{l}
\left.\frac{\partial \boldsymbol{\varepsilon}_{o j}^{T}\left(\boldsymbol{\varepsilon}_{o i}, \boldsymbol{\beta}_{o i j}\right)}{\partial \boldsymbol{\varepsilon}_{o i}}\right|_{0} \\
\left.\frac{\partial \boldsymbol{\varepsilon}_{o j}^{T}\left(\boldsymbol{\varepsilon}_{o i}, \boldsymbol{\beta}_{o i j}\right)}{\partial \boldsymbol{\beta}_{o i j}}\right|_{0}
\end{array}\right]+\left[\begin{array}{ll}
\left.\frac{\partial^{2}\left[U_{j, \varepsilon}^{T} \boldsymbol{\varepsilon}_{o j}\left(\boldsymbol{\varepsilon}_{o i}, \boldsymbol{\beta}_{o i j}\right)\right]}{\partial \boldsymbol{\varepsilon}_{o i} \partial \boldsymbol{\varepsilon}_{o i}^{T}}\right|_{0} & \left.\frac{\partial^{2}\left[U_{j, \varepsilon}^{T} \boldsymbol{\varepsilon}_{o j}\left(\boldsymbol{\varepsilon}_{o i}, \boldsymbol{\beta}_{o i j}\right)\right]}{\partial \boldsymbol{\varepsilon}_{o i} \partial \boldsymbol{\beta}_{o i j}^{T}}\right|_{0} \\
\left.\frac{\partial^{2}\left[U_{j, \varepsilon}^{T} \boldsymbol{\varepsilon}_{o j}\left(\boldsymbol{\varepsilon}_{o i}, \boldsymbol{\beta}_{o i j}\right)\right]}{\partial \boldsymbol{\beta}_{o i j} \partial \boldsymbol{\varepsilon}_{o i}^{T}}\right|_{0} & \left.\frac{\partial^{2}\left[U_{j, \varepsilon}^{T} \boldsymbol{\varepsilon}_{o j}\left(\boldsymbol{\varepsilon}_{o i}, \boldsymbol{\beta}_{o i j}\right)\right]}{\partial \boldsymbol{\beta}_{o i j} \partial \boldsymbol{\beta}_{o i j}^{T}}\right|_{0}
\end{array}\right] \\
& =\left[{ }^{o j} B_{o i} \quad{ }^{o j} B_{\text {Loij }} D_{\text {Loij }}\right]^{T} U_{j, \varepsilon \varepsilon}\left[{ }^{o j} B_{o i} \quad{ }^{o j} B_{\text {Loij }} D_{\text {Loij }}\right]- \\
& {\left[\begin{array}{lll}
0_{6 \times 3} & { }^{o j} B_{o i} I_{3 \xi}^{T} & 0_{6 \times 5}
\end{array}\right]^{T}\left[\begin{array}{cc}
0_{3 \times 3} & 0_{3 \times 3} \\
{\left[\left(I_{3 x} U_{j, \varepsilon}\right) \otimes\right.} & 0_{3 \times 3}
\end{array}\right]\left[\begin{array}{lll}
0_{6 \times 3} & { }^{o j} B_{o i} I_{3 \xi}^{T} & { }^{o j} B_{L o i j} D_{L o i j}
\end{array}\right]+}
\end{aligned}
$$

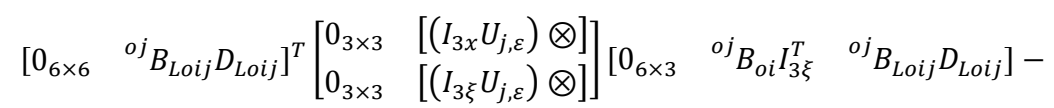

$$
\begin{aligned}
& {\left[\begin{array}{lll}
0_{6 \times 6} & { }^{o j} B_{L o i j}\left[C_{o i j}, \boldsymbol{u}_{r z}\right] & 0_{6 \times 2}
\end{array}\right]^{T}\left[\begin{array}{cc}
0_{3 \times 3} & {\left[\left(I_{3 x} U_{j, \varepsilon}\right) \otimes\right.}
\end{array}\right]\left[\begin{array}{ll}
\left.\left(I_{3 x} U_{j, \varepsilon}\right) \otimes\right] & {\left[\left(I_{3 \xi} U_{j, \varepsilon}\right) \otimes\right.}
\end{array}\right]\left[\begin{array}{ll}
0_{6 \times 6} & \left.{ }^{o j} B_{L o i j} D_{L o i j}\right]+
\end{array}\right.}
\end{aligned}
$$

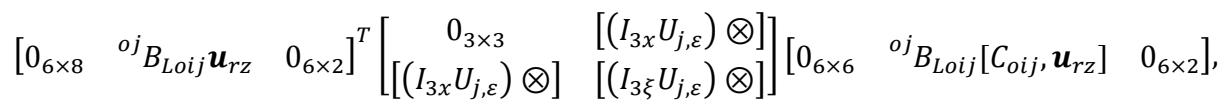

where

$$
U_{j, \varepsilon}:=\left.\frac{\partial U_{j}\left(\varepsilon_{o j}\right)}{\partial \varepsilon_{o j}}\right|_{0}, U_{j, \varepsilon \varepsilon}:=\left.\frac{\partial^{2} U_{j}\left(\varepsilon_{o j}\right)}{\partial \varepsilon_{o j} \partial \varepsilon_{o j}^{T}}\right|_{0}
$$

